\title{
Protein 0-Mannosyltransferases Affect Sensory Axon Wiring and Dynamic Chirality of Body Posture in the Drosophila Embryo
}

\author{
Ryan Baker, ${ }^{\star}$ Naosuke Nakamura, ${ }^{\star}$ - Ishita Chandel, ${ }^{\star}$ B Brooke Howell, $\odot$ Dmitry Lyalin, and $\odot$ Vladislav M. Panin \\ Department of Biochemistry and Biophysics, Texas A\&M University, College Station, Texas 77843-2128
}

Genetic defects in protein 0-mannosyltransferase 1 (POMT1) and POMT2 underlie severe muscular dystrophies. POMT genes are evolutionarily conserved in metazoan organisms. In Drosophila, both male and female POMT mutants show a clockwise rotation of adult abdominal segments, suggesting a chirality of underlying pathogenic mechanisms. Here we described and analyzed a similar phenotype in POMT mutant embryos that shows left-handed body torsion. Our experiments demonstrated that coordinated muscle contraction waves are associated with asymmetric embryo rolling, unveiling a new chirality marker in Drosophila development. Using genetic and live-imaging approaches, we revealed that the torsion phenotype results from differential rolling and aberrant patterning of peristaltic waves of muscle contractions. Our results demonstrated that peripheral sensory neurons are required for normal contractions that prevent the accumulation of torsion. We found that POMT mutants show abnormal axonal connections of sensory neurons. POMT transgenic expression limited to sensory neurons significantly rescued the torsion phenotype, axonal connectivity defects, and abnormal contractions in POMT mutant embryos. Together, our data suggested that protein 0-mannosylation is required for normal sensory feedback to control coordinated muscle contractions and body posture. This mechanism may shed light on analogous functions of POMT genes in mammals and help to elucidate the etiology of neurological defects in muscular dystrophies.

Key words: axon patterning; chirality; Drosophila; muscle contractions; protein 0-mannosylation; sensory neurons

Significance Statement

Protein 0-mannosyltransferases (POMTs) are evolutionarily conserved in metazoans. Mutations in POMTs cause severe muscular dystrophies associated with pronounced neurological defects. However, neurological functions of POMTs remain poorly understood. We demonstrated that POMT mutations in Drosophila result in abnormal muscle contractions and cause embryo torsion. Our experiments uncovered a chirality of embryo movements and a unique POMT-dependent mechanism that maintains symmetry of a developing system affected by chiral forces. Furthermore, POMTs were found to be required for proper axon connectivity of sensory neurons, suggesting that 0 -mannosylation regulates the sensory feedback controlling muscle contractions. This novel POMT function in the peripheral nervous system may shed light on analogous functions in mammals and help to elucidate pathomechanisms of neurological abnormalities in muscular dystrophies.

\section{Introduction}

Protein O-mannosylation (POM) represents a prominent example of protein glycosylation with crucial biological functions

Received Feb. 6, 2017; revised Nov. 1, 2017; accepted Nov. 6, 2017.

Author contributions: R.B., N.N., I.C., and V.M.P. designed research; R.B., N.N., I.C., B.H., D.L., and V.M.P. performed research; R.B., N.N., I.C., B.H., D.L., and V.M.P. analyzed data; R.B., N.N., I.C., and V.M.P. wrote the paper.

The project was supported in part by National Institutes of Health (NIH) Grants NS-099409, NS075534, and CONACYT 2012-037(S) to V.M.P. Stocks obtained from the Bloomington Drosophila Stock Center at Indiana University (NIH Grant P40-OD-018537) were used in this study. We thank Graeme Davis, Eric Olson, and Cynthia Hughes for providing transgenic Drosophila strains. We also thank Aaron Henrichsen (Penn State University) for his help with statistical analyses. In addition, we thank Paul Hardin, Bruce Riley, and Ginger Carney for valuable discussions and comments on the manuscript.

*R.B., N.N., and I.C. contributed equally to this work.

The authors declare no competing financial interests.
(Moremen et al., 2012; Wells, 2013; Yoshida-Moriguchi and Campbell, 2015). Two protein O-mannosyltransferases (POMTs) that mediate POM, POMT1 and POMT2, are conserved in all animals. Enzymatic POM activity requires the formation of a complex between POMT1 and POMT2 that functions in the endoplasmic reticulum (ER). POM affects the development and physiology of mammalian muscular and nervous systems, while genetic inactivation of POMT1 or POMT2 results in early embryonic lethality in

Correspondence should be addressed to Vladislav M. Panin, Department of Biochemistry and Biophysics, Texas A\&M University, TAMU 2128, College Station, TX 77843-2128. E-mail: panin@tamu.edu.

D0I:10.1523/JNEUROSCI.0346-17.2017

Copyright $\odot 2018$ the authors $\quad 0270-6474 / 18 / 381850-16 \$ 15.00 / 0$ 
mice (Willer et al., 2004; Martin, 2007; Hu et al., 2011; Freeze et al., 2015).

The best characterized O-mannosylation target is dystroglycan (Dg), a glycoprotein that serves as a cell membrane-spanning bridge linking the ECM and cytoskeleton (for review, see Barresi and Campbell, 2006; Yoshida-Moriguchi and Campbell, 2015). Defects in POM compromise interactions between Dg and its extracellular ligands, which causes congenital muscular dystrophies termed dystroglycanopathies (Godfrey et al., 2011). These dystrophies are associated with neurological abnormalities, including brain developmental defects, which indicates that POM has important functions in neural development (Mercuri and Muntoni, 2013). Recent studies have revealed that POM is present on many glycoproteins that can function in the nervous system, including cadherins, plexins, and receptor protein tyrosine phosphatase $\zeta$ (RPTP $\zeta$; Vester-Christensen et al., 2013; Dwyer et al., 2015). Experiments in mouse models indicated that POM is required for integrity of the pial basement membrane and neuronal migration (Hu et al., 2011; Li et al., 2011). However, most studies have concentrated on POM-mediated regulation of Dg activity in muscles, while POM functions in the nervous system remain poorly understood.

Significant sequence conservation between Drosophila and mammalian POMTs suggests that POMT functions are conserved in animal evolution, from insects to mammals (MartínBlanco and García-Bellido, 1996; Ichimiya et al., 2004; Lyalin et al., 2006). Indeed, previous studies demonstrated that Drosophila POMT1 and POMT2 [rotated abdomen ( $r t)$ and twisted (tw), respectively] show strong synergistic genetic interactions (Ichimiya et al., 2004; Lyalin et al., 2006). RT and TW proteins colocalize in the ER, and their simultaneous activity is required for O-mannosylation of Drosophila $\mathrm{Dg}$ in vitro and in vivo, indicating that $\mathrm{RT}$ and TW, similar to their mammalian counterparts, function together as a heteromeric enzymatic complex (Lyalin et al., 2006; Nakamura et al., 2010a). Mutations in $r t, t w$, and $D g$ result in muscle developmental defects, abnormal synaptic transmission, and age-dependent muscle degeneration (Martín-Blanco and GarcíaBellido, 1996; Haines et al., 2007; Shcherbata et al., 2007; Wairkar et al., 2008; Ueyama et al., 2010), the phenotypes also associated with human dystroglycanopathies (Mercuri and Muntoni, 2013). However, the pathogenic mechanisms in Drosophila POMT mutants are poorly understood. Interestingly, $r t$ and $t w$ mutants show misalignment of adult abdominal segments that causes the clockwise "abdomen rotation" phenotype, a unique example of a developmental defect associated with abnormal chirality (MartínBlanco and García-Bellido, 1996; Lyalin et al., 2006). This phenotype was discovered in the Morgan laboratory in 1918 (Bridges and Morgan, 1923), yet its underlying mechanism still remains unexplained. Here we described a novel POMT embryonic phenotype associated with body torsion that is reminiscent of the abdomen rotation in adults. The torsion phenotype arises during peristaltic muscle contractions. Our analyses uncovered a directional rolling behavior of embryos inside the eggshell, which revealed a novel asymmetry in Drosophila development. We found that $P O M T$ mutations affect peripheral sensory neurons, coordination of muscle contractions, and dynamic chirality of body torsion caused by embryo rolling. Based on these data, we proposed a mechanism that explains how the defect in muscle contractions may cause the torsion phenotype.

\section{Materials and Methods}

Drosophila stocks and crosses. The mutant alleles for $r t$ and $t w$ were previously described, as follows: $t w^{I}$ is a hypomorphic allele; $r t^{2}, r t^{p}$, and $r t^{571}$ are strong hypomorphic alleles that are close to amorphs (MartínBlanco and García-Bellido, 1996; Lyalin et al., 2006); sens ${ }^{E 2}$ is a loss-offunction allele (Nolo et al., 2000); and $D g^{086}$ is a null allele (Christoforou et al., 2008). Several transgenic insertions were obtained from different researchers: MHC-GAL4 was from Graeme Davis; DMEF2-GAL4 was received from Eric Olson; and MHC-GFP line was a gift from Cynthia Hughes. UAS- $t w$ transgene was previously described (Lyalin et al., 2006). Other mutant and transgenic strains were obtained from the Bloomington Drosophila Stock Center at Indiana University. Unless indicated otherwise, embryos of either sex were included in all analyses, as our experiments, as well as studies by other groups, revealed no evidence of sex-specific phenotypes of POMT mutants.

Fluorescent staining and microscopy. Embryos were dechorionated, fixed, and dissected manually from the vitelline membrane according to published protocols (Rothwell and Sullivan, 2000). They were stained with Alexa Fluor 488-conjugated phalloidin (Invitrogen) using 1:200 dilution. Digital images were obtained using a Zeiss Axioplan 2 fluorescent microscope with the ApoTome module for optical sectioning. AxioVision and ImageJ software were used for $3 \mathrm{D}$ reconstruction and $Z$-projections of fluorescent samples.

First instar larvae expressing tdTomato or tdGFP in the Ppk pattern (Han et al., 2012; Yang et al., 2014), in a wild-type, $t w$, or $r t$ mutant background, were collected. The CNS (brain lobes together with the ventral ganglion) was dissected, fixed in PBS with $4 \%$ paraformaldehyde, and stained with rabbit anti-dsRed or anti-GFP antibody (Clontech) in a 1:1000 dilution. Mouse anti-Fasciclin II (FasII) and anti-Repo antibodies [1D4 and 8D12 from Developmental Studies Hybridoma Bank (DSHB), respectively] were used in a 1:10 dilution. Alexa Fluor 546-conjugated goat anti-rabbit or anti-mouse antibodies were used as a secondary antibody in a 1:250 dilution. Stained samples were mounted on slides and imaged using the Zeiss Axioplan 2 Microscope using $40 \times$ magnification objective. To minimize potential errors, control and experimental samples were stained using the same master-mix of antibodies and imaged with the same settings for camera, illumination, and microscope. Commissural branch thickness was measured in stacks of optical sections with $1 \mu \mathrm{m}$ step size using ImageJ for every branch in a given ventral nerve cord and averaged, and the collective average from a given genotype reported. Images were analyzed blindly without genotype information. Longitudinal tracts were scored by averaging the brightness of the spaces between commissural branches for a background brightness, and tracts were scored as "absent" when the brightness of the region where the tract was expected to be was $<10 \%$ above background, as measured by ImageJ.

Flp-out analysis of individual axons. A Flp-out approach for labeling individual class IV dendritic arborization (da) neurons was performed using previously described strategy (Grueber et al., 2007) with some modifications. Briefly, males of $y w h s F l p ;+; r t^{P} / / T(2 ; 3) T S T L 14$, SM5: $T M 6 B, T b^{1}$ were crossed to 10XUAS $>$ (FRT stop) $>$ GFP-Myr; $r t^{2} p p k G a l 4 /$ TM3-KrGFP females. F1 progeny was collected as embryos for 17-20 h and given a heat shock at $34^{\circ} \mathrm{C}$ for $12 \mathrm{~min}$ to induce the expression of Flp recombinase. Then these embryos were allowed to develop until third instar at $25^{\circ} \mathrm{C}$, and brains of $y w h s F l p / 10 X U A S>($ FRT stop $)>G F P-M y r$; $r t^{P} / r t^{2} p p k G a l 4$ larvae were dissected, fixed, and incubated with primary mouse anti-GFP antibody (8H11, DSHB) at 1:100 dilution, followed by incubation with secondary goat anti-mouse Alexa Fluor 488 (Invitrogen) antibody diluted 1:250. For wild-type control, the same genotype, but without $r t$ mutation, was analyzed. Stained brains were mounted in Vectashield (Vector Laboratories) and imaged using a Zeiss Axio Imager Fluorescence Microscope with ApoTome module for optical sectioning.

Live imaging of embryos. A GFP-tagged myosin heavy chain (MHC) was expressed in wild-type, POMT mutant, and senseless mutant backgrounds. Embryos were staged according to (Pereanu et al., 2007). Stage 17 embryos with an air-filled trachea and no obvious midline misalignment were collected and placed on a slide with the dorsal appendages up. Muscle contractions were recorded using a Hamamtsu ORCA-Flash4.0 CMOS digital camera for $1-2 \mathrm{~h}$ using an X-Cite BDX LED with an emission maximum of $460 \mathrm{~nm}$ and an ET525/50 emission filter on a Zeiss Examiner D1 microscope. Videos were analyzed using ImageJ software. Rolling was assessed by measuring the average displacement of tracheal dorsal trunks produced by individual contractions. The dorsal midline 
A

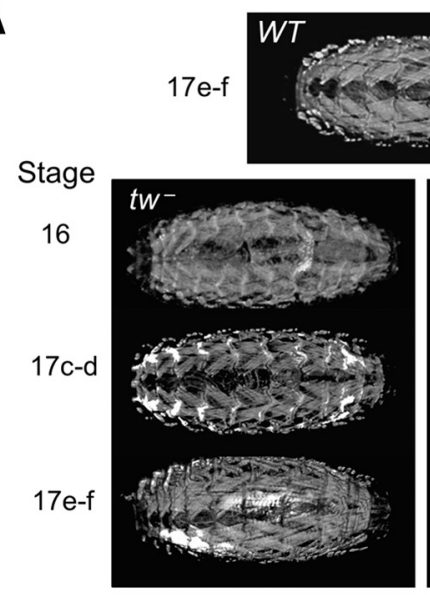

C

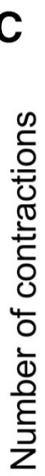

0
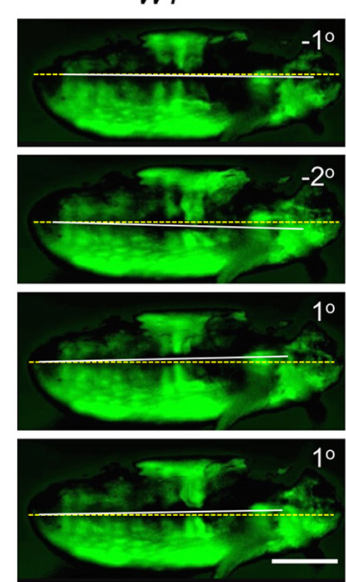

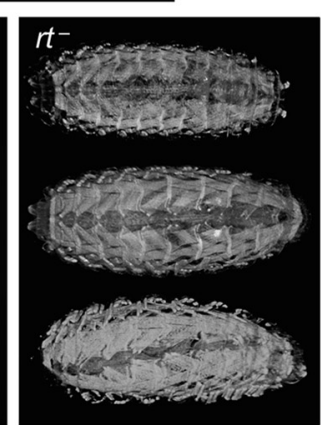

$r t^{-}$
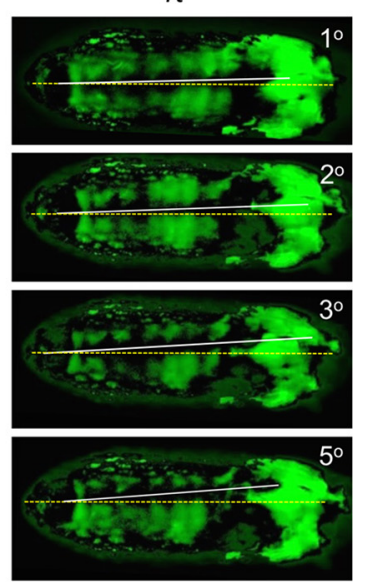

B

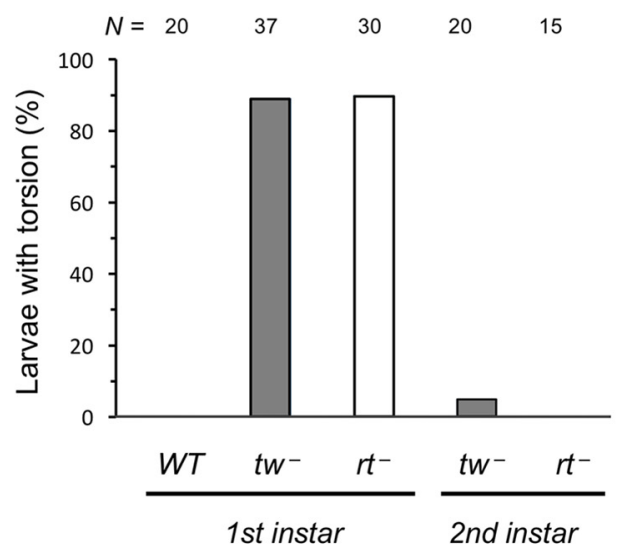

D

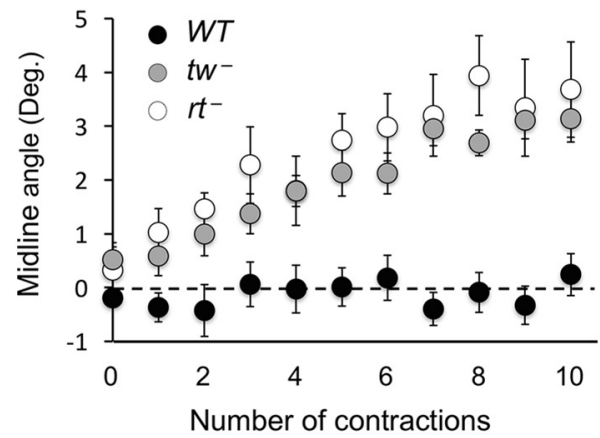

Figure 1. Body torsion phenotype of POMT mutant embryos. A, POMT mutants show body torsion phenotype. Top, Wild-type embryos have no torsion at any stage (stage $17 \mathrm{e}-\mathrm{f}$ is shown). Bottom, $t w$ and $r$ mutants show no torsion at stage 16 and usually no torsion at stages $17 \mathrm{a}-\mathrm{d}$. By stages $17 \mathrm{e}-\mathrm{f}$, nearly $100 \%$ of mutant embryos show left-handed torsion. $\boldsymbol{B}$, The torsion phenotype is highly penetrant in first instar larvae but essentially disappears in the second instar stage. $N$, number of larvae analyzed for each stage. C, Representative images displaying changes in dorsal midline (white line) angle over several contractions. Dashed yellow line represents the AP axis. Positive and negative angles between yellow and white lines represent left-handed and right-handed torsion, respectively. In $\boldsymbol{A}$ and $\boldsymbol{C}$, anterior is to the right. $\boldsymbol{D}$, Average angle between dorsal midline and the AP axis over the first 10 contractions. Ten embryos were analyzed for $W T$ and $t w^{-}$, and 5 embryos were analyzed for $r t^{-}$. Scale bars, $100 \mu \mathrm{m}$. WT, wild-type. $r t^{-}, r t^{P} / r t^{P} . t w^{-}, t w^{7} / t w^{7}\left(o r t w^{7} / Y\right)$. All genotypes also included a transgenic MHC-GFP construct to visualize muscles. Statistical analyses: error bars show SEM; starting at contraction 5 , the difference between WT and either mutant genotype is statistically significant (ANOVA with a Tukey-HSD post hoc test: largest $p$ values are $0.02,0.02,6 \mathrm{E}-5,6 \mathrm{E}-5,1 \mathrm{E}-3$, and $1 \mathrm{E}-4$ for contractions $5-10$, respectively).

angle was measured by selecting points halfway between left and right dorsal muscles, drawing a line connecting those points, and measuring its angle relative to the anterior-posterior (AP) axis. The position of the AP axis was estimated based on the position of the anterior and posterior tips of the embryo between contractions waves, when muscles do not show significant activity. Although embryos can slightly wiggle inside the embryo shell when muscles contract, in addition to rolling, they cannot significantly change their position because chorionic layers of the shell are rigid and the shell closely reflects the shape and volume of the embryo between muscle contractions. The posterior and anterior tips of the embryo are always located at posterior and anterior tips of the shell between muscle contractions, and thus the AP axis of the shell provides a truthful approximation of the embryo AP axis between contractions. Embryos with an absolute value of dorsal midline angle of $>2^{\circ}$ were scored as having torsion as the difference between wild-type and mutant embryos become statistically significant at this point (Fig. 1D). Embryos were scored as having no torsion when this angle was $<2^{\circ}$. Left-handed and right-handed torsion phenotypes corresponded to positive and negative values of the dorsal midline angle, respectively.

For GFP intensity analysis, cross sections $5 \mu \mathrm{m}$ wide and $25 \mu \mathrm{m}$ apart each, were selected along the anterior-posterior body axis. A baseline for each cross section was established as the average intensity during the time between contraction waves. Muscle-shortening amplitude was estimated by an increase in GFP intensity that occurs as muscle contractions bring more GFP into the vicinity of focal area. The most anterior and posterior cross sections, as well as a cross section halfway between anterior and posterior ends of the embryo, were used for assessment of muscle contraction amplitude. Integrated fluorescence intensity was measured for every frame of each cross section and normalized to the baseline. Anterior intensity was divided by posterior intensity to produce a ratio.

UAS-GAL4-mediated rescue experiments and embryonic torsion scoring. In experiments investigating the spatial requirements of POMT activity, the expression of a UAS- $t w$ transgenic construct (Lyalin et al., 2006) was induced with each of the drivers described. All driver-mediated rescue experiments included controls, confirming that neither GAL4 drivers alone nor UAS-tw alone influence the mutant phenotype. All flies were reared at $25^{\circ} \mathrm{C}$ in a light- and humidity-controlled environment, as described previously (Islam et al., 2013).

Experimental design and statistical analysis. Experiments were performed at least three times. $N$ refers to the number of embryos or larvae of particular genotypes (Fig. 1; see also Fig. 4) or the number of individual axons with particular morphology (see Fig. 6). Statistical analyses were performed by one-way ANOVA with Tukey's HSD post hoc comparisons for significance. For bar graphs and box plots, data for each bar 
or box in the graph were analyzed as a separate column. For the line graph in Figure $1 D$, data for each contraction number were compared across the three genotypes. In all figures, 1 and 2 asterisks represent $p$ values of $<0.05$ and 0.01 , respectively; NS indicates that no significant differences were found $(p>0.05)$. Details on statistical analysis are included in figure legends and text.

\section{Results \\ POMT mutant embryos accumulate body torsion during peristaltic muscle contractions}

Previous studies of $r t$ and $t w$ mutants have mostly concentrated on adult and larval stages, while the effect of POMT mutations on embryonic development has not been well characterized (MartínBlanco and García-Bellido, 1996; Ichimiya et al., 2004; Haines et al., 2007; Ueyama et al., 2010). With the rationale that potential phenotypes of POMT mutants at early developmental stages could shed light more directly on pathological mechanisms associated with POM defects, we decided to focus on embryonic stages. We analyzed $r t$ and $t w$ mutant embryos and discovered that they have a prominent body posture defect manifested as a whole-body left-handed torsion (rotation of more anterior segments, relative to more posterior ones, in counterclockwise direction, as viewed from the posterior), which is reminiscent of the adult abdomen rotation phenotype (Fig. 1A). This "embryonic torsion" phenotype becomes pronounced at stage 17, the last stage of embryonic development. The penetrance of the phenotype in $r t^{2 / 571}$ mutant embryos was found to be $32 \%(N=19)$ at an early phase of stage 17 (stages $17 \mathrm{a}-\mathrm{d})$, and $100 \%(N=24)$ at its last phase (stages 17e-f; stages are according to the study by Pereanu et al., 2007). This torsion persisted into the first instar larval stage with $90 \%$ penetrance but was almost entirely absent in second instar larvae. Similar results were obtained for $t w^{1} / t w^{1}$ mutants (Fig. 1B). No posture defects or any conspicuous muscle abnormalities were observed in stage 16 embryos $(N=84$; Fig. $1 A)$. Since the somatic musculature and epidermis are already formed at stage 16, these data suggested that the torsion phenotype may originate from abnormal muscle contractions rather than from a developmental defect of musculature or abnormal epidermal morphology. This scenario is also consistent with the fact that the torsion phenotype becomes prominent soon after the embryos initiate coordinated muscle contractions (Pereanu et al., 2007; Crisp et al., 2008).

To test more directly whether muscle contractions are involved in generating the embryo torsion phenotype, we used a live-imaging approach to analyze muscle contractions in embryos with muscles labeled by transgenically expressed MHC-GFP (Hughes and Thomas, 2007). Once embryos initiated peristaltic contractions, we measured the angle of the embryo dorsal midline, defined by equidistant position from muscles on the left and right sides of the embryo, relative to the AP axis before and after each contraction. We found that during embryonic contractions the midline remained essentially parallel to the AP axis in wild-type embryos, even though the midline could change its position within the shell when embryos undergo contractions. In mutants, the midline accumulated an angle, which revealed increasing left-handed torsion of embryos (Fig. 1C,D; Movies 1, 2, 3). These results demonstrated that the embryonic body torsion defect arises in mutants during peristaltic muscle contractions and also revealed a correlation between the increase of torsion and the number of contraction waves generated by mutant embryos, which suggested that contraction waves could induce torsion in mutant embryos.

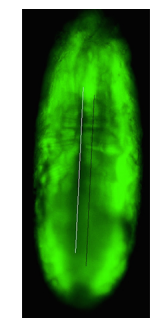

Movie 1. Effect of contractions on the position of dorsal midline in wild-type embryos. Representative example of eight consecutive contraction waves. Over the course of several contractions, the dorsal midline moves but remains essentially parallel to the AP axis. The video includes a time series of images taken at 4 frames/s (fps) and shown at the frame rate of $20 \mathrm{fps}$. Transgenic MHC-GFP expression was used to

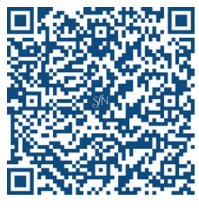
visualize muscle contractions. Black and white lines indicate position of the midline before and after contractions, respectively.

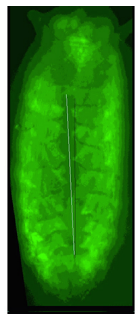

Movie 2. Effect of contractions on dorsal midline in tw mutants. Over the course of several contractions the anterior portion of the midline moves to the left further than the posterior portion, resulting in body torsion. Genotype, $t w^{1} / t w^{1}$ (or $t w^{1} / M$ ). The video shows 9 contraction waves recorded at 4 frames/s ( $\mathrm{fps}$ ) and shown at a frame rate of $20 \mathrm{fps}$. Transgenic MHC-GFP expression was used to visualize muscle contractions. Black and white lines indicate the position of the midline before and after contractions, respectively.

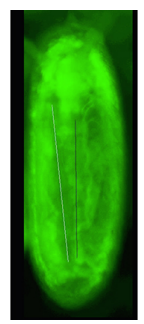

Movie 3. Effect of contractions on $\mathrm{rt}^{-}$dorsal midline. As in $\mathrm{tw}^{-}$, over the course of several contractions the anterior portion of the midline moves to the left farther than the posterior portion, resulting in torsion. Genotype, $r t^{P} / r t^{P}$. The time series of images was recorded and sped up as in other movies. Transgenic MHC-GFP expression was used to visualize muscle contractions. Black and white lines indicate the position of the midline before and after contractions, respectively.

\section{Peristaltic muscle contractions induce chiral embryonic} rolling within the eggshell

Before investigating further the link between contraction waves and the embryo torsion phenotype, we decided to characterize in more detail how embryos behave during peristaltic contractions. In both wild-type and POMT mutant embryos, peristaltic waves of contractions can propagate from posterior to anterior (forward waves) or from anterior to posterior (backward waves) ends of the embryo. We first focused on analyzing these waves in wildtype embryos. Upon close inspection of contraction waves, we discovered that the embryo rolls within its shell during these 
A

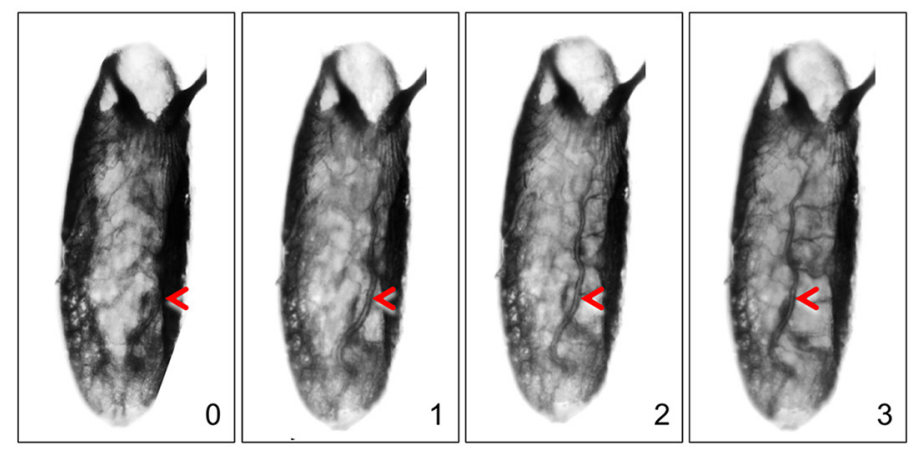

Forward contractions

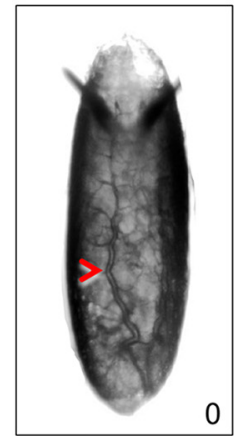

0
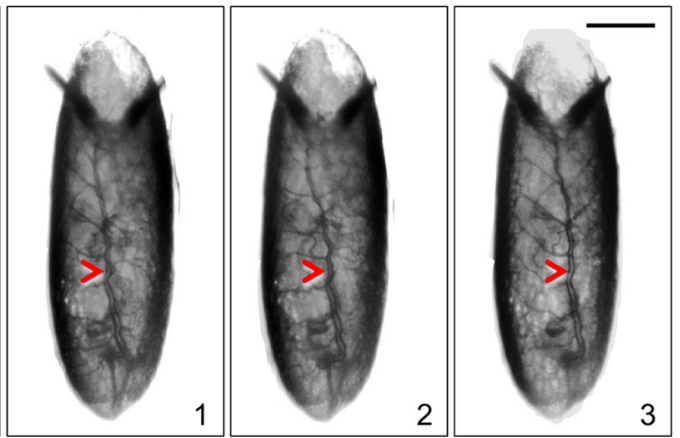

Backward contractions
B

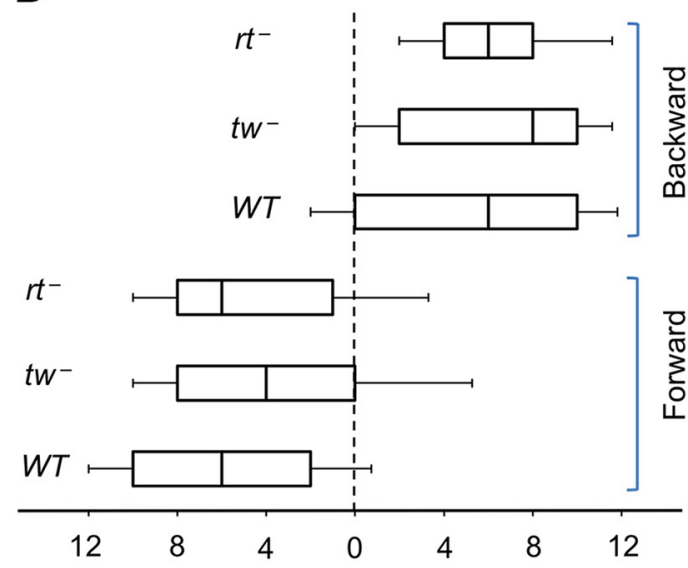

Left Distance rolled $(\mu m)$

Right

C
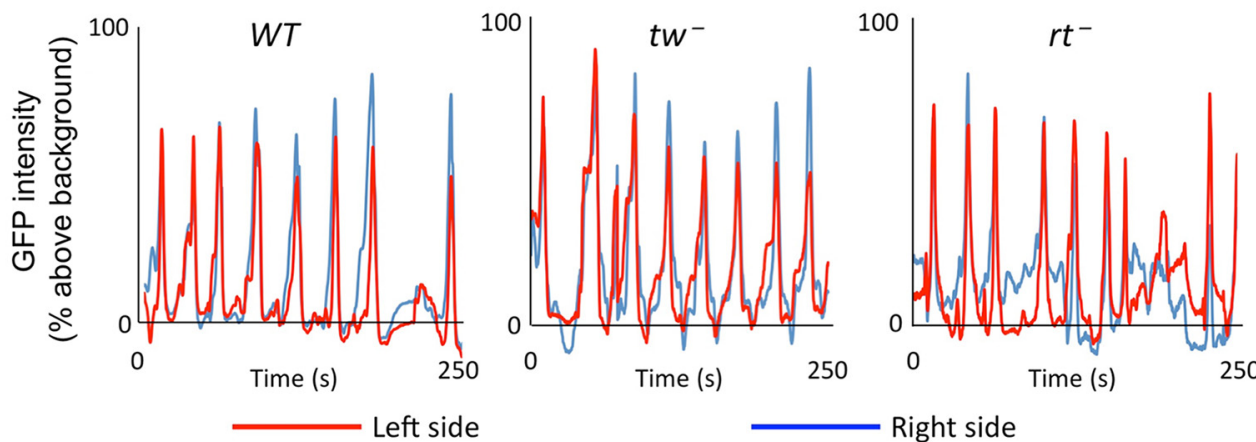

D
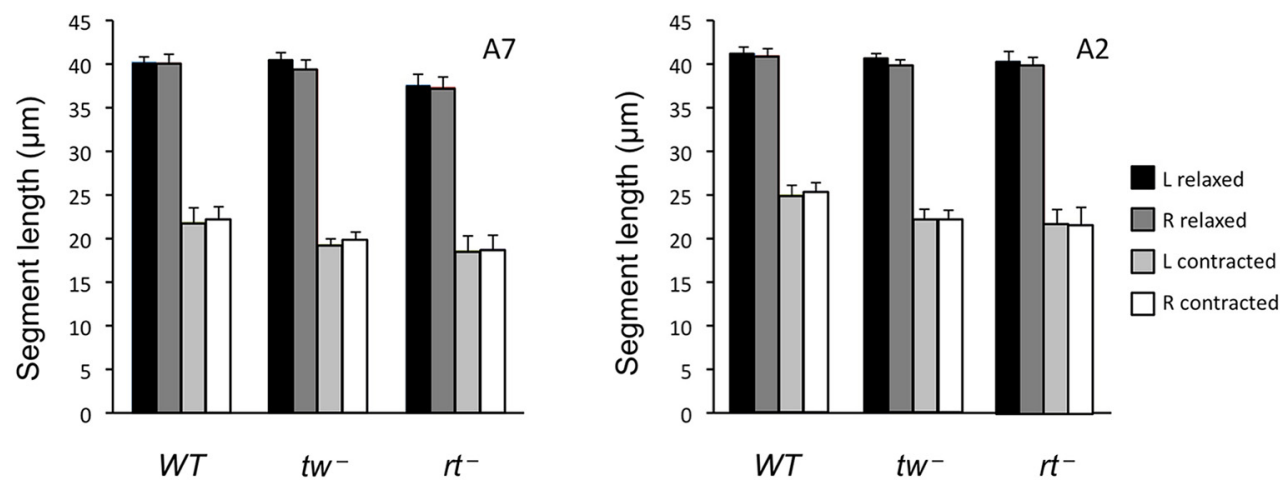

Figure 2. Embryos roll chirally inside their shells during peristaltic contractions. $\boldsymbol{A}$, Time series of rolling during consecutive forward and backward contractions. Rolling is obvious from changes in the position of a dorsal trunk trachea (red arrowhead). Scale bars, $100 \mu \mathrm{m}$. $\boldsymbol{B}$, Quantification of the average distance rolled during backward and forward contraction waves in WT, $t w^{-}$, and $r t^{-}$ embryos, as measured from halfway between anterior and posterior ends. Boxes represent the interquartile range, lines represent the median values, and error bars are SEs of the mean. No significant difference was detected among genotypes for either forward or backward rolling. Number of embryos and contractions analyzed (for each type of contractions): 10 embryos and at least 45 contractions per genotype for WT and tw ${ }^{-}$, and 5 embryos and at least 21 contractions for $r t^{-}$. C, D, Mutant embryos do not have left-right contraction (Figure legend continues.) 


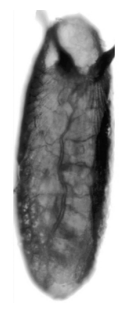

Movie 4. Embryos roll to the left during forward contractions. Representative example of leftward rolling during forward waves of contractions. This phenomenon occurs in all genotypes tested. Wild-type embryo is shown. The time series of images was recorded and sped up as in other movies.

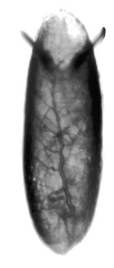

Movie 5. Embryos roll to the right during backward contractions. Representative example of rightward rolling during backward embryonic contractions. This phenomenon occurs in all genotypes tested. Wild-type embryo is shown. Time series of images was recorded and sped up as in other movies.

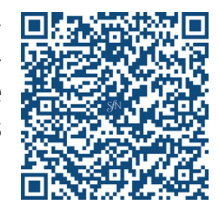

Embryo torsion arises during abnormal alteration of muscle contraction waves

Wild-type embryos could roll over the course of several contractions while maintaining a straight body posture and having their dorsal midline essentially parallel to the embryo AP axis. POMT mutants, on the other hand, developed torsion as their anterior and posterior segments rolled differently and the dorsal midline accumulated an angle relative to the AP axis. A recent study demonstrated that damage of Eve interneurons could cause left-right (LR) asymmetry of muscle contractions and larval crawling defects (Heckscher et al., 2015), suggesting that a related pathomechanism might cause torsion in POMT mutant embryos. However, when we analyzed mutants, we could not find any significant left-right asymmetry of muscle contractions as the timing and amplitude of contraction on the left and right sides of the embryo were not significantly different, and mutants were not different from wild-type controls (Fig. 2C,D). These results, together with the fact that defects in Eve interneurons result in randomized rather than biased asymmetry in muscle coordination, suggested that a different mechanism underlies the embryo torsion phenotype.

We alternatively hypothesized that "differential rolling" of mutant embryo segments could play a part in producing torsion. Such differential rolling could arise from differences in the force that induce the rolling of different segments and depend on the amplitude of contractions at a particular segment. This assumption is consistent with the fact that rolling strictly correlates with contractions as it is induced only upon wave propagation but not between the waves. This hypothesis predicts that differential rolling can be associated with differences in contraction amplitude as a wave propagates along the embryo. Furthermore, since lefthanded torsion in mutants is associated with greater rolling at the end of the wave, it is expected that mutants should show an increasing strength of contractions along wave propagation. We tested these predictions by analyzing single waves simply propagating from one end to the other, which we designated here as type 1 waves (Fig. 3A,B; Movie 6; also see discussion below). Surprisingly, we found that these waves decrease in amplitude on average as they progress in mutant as well as wild-type embryos, regardless of their direction of propagation (Fig. $3 C, D$ ). Thus, the differential rolling mechanism by itself is not able to explain the left-handed torsion of mutants. Moreover, assuming that rolling positively correlates with contraction amplitude, these simple waves are predicted to result in the accumulation of net righthanded body torsion in both mutant and wild-type genotypes. Indeed, when we correlated contractions with the change in the angle of the midline, we found that type 1 waves correspond to accumulation of right-handed torsion on average in both wildtype and POMT mutant genotypes, regardless of whether the wave propagated in the forward or reverse direction (Fig. $3 E$ ). These data seemingly contradicted the fact that over the course of multiple waves wild-type embryos maintain a straight body posture while mutants accumulate a left-handed torsion. To resolve this apparent conundrum, we performed a more detailed analysis of the pattern of contraction waves, which revealed that embryos occasionally generated a biphasic, rather than a simple, wave. During such waves (designated herein as type 2 waves) contractions initiated as a partial backward wave, which propagated until halting momentarily as a static overcontraction of a broad posterior region. This overcontraction did not relax until it was swept by the second phase, which propagated forward from the posterior end, often with increasing amplitude (Fig. $3 A, B$; Movie 7). Importantly, type 2 waves correlated with a change in the midline 
A

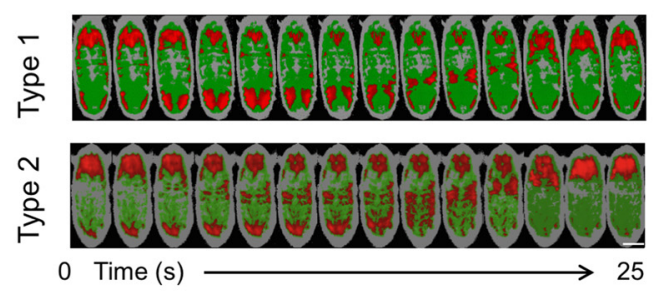

C

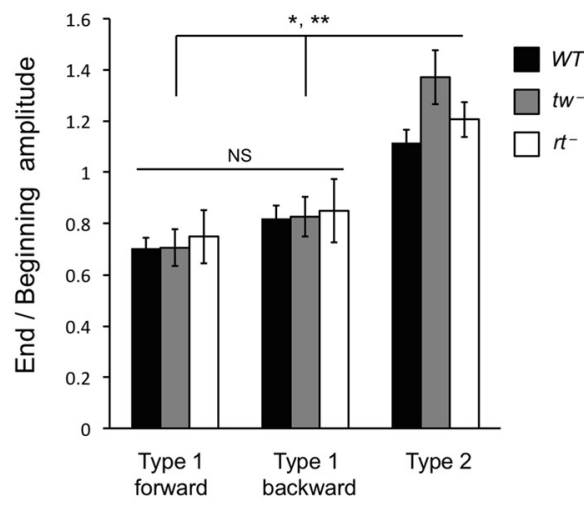

E

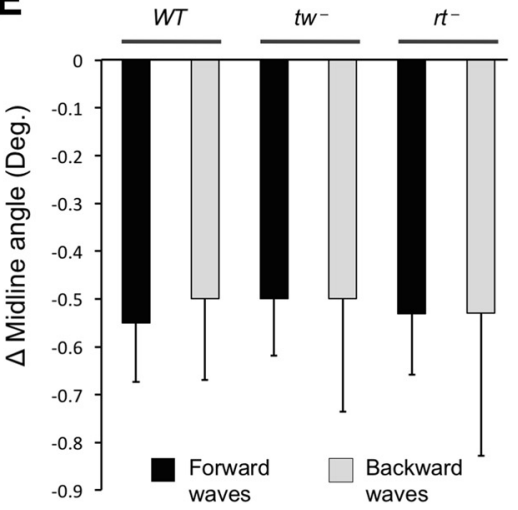

B

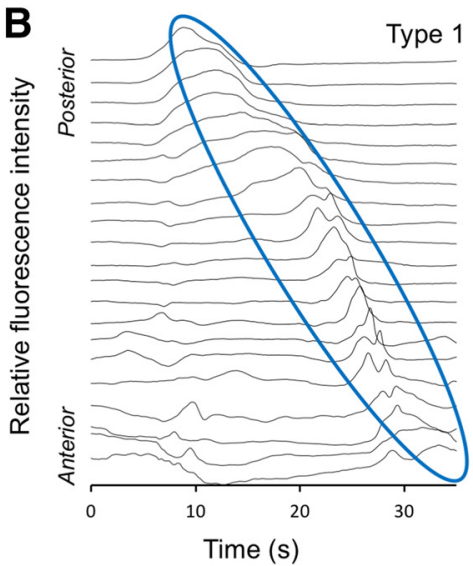

D

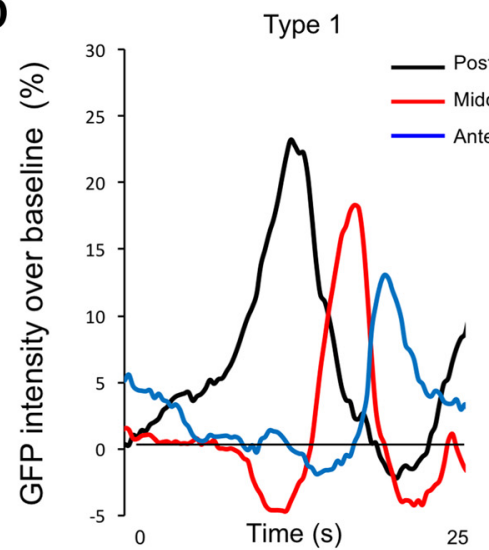

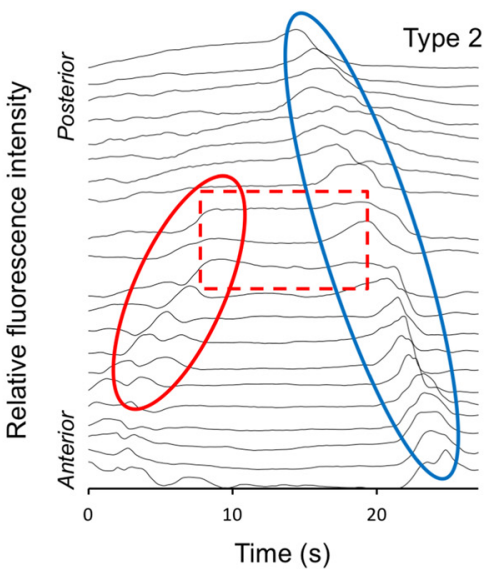

Type 2

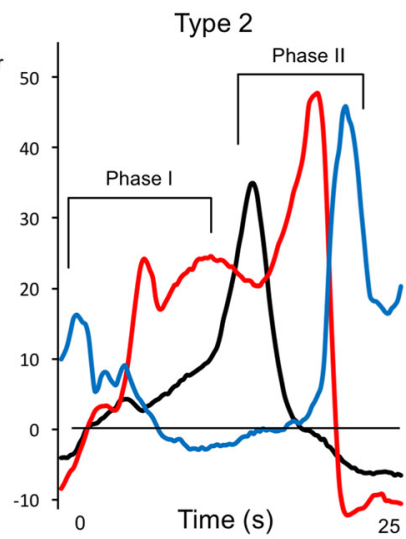

F

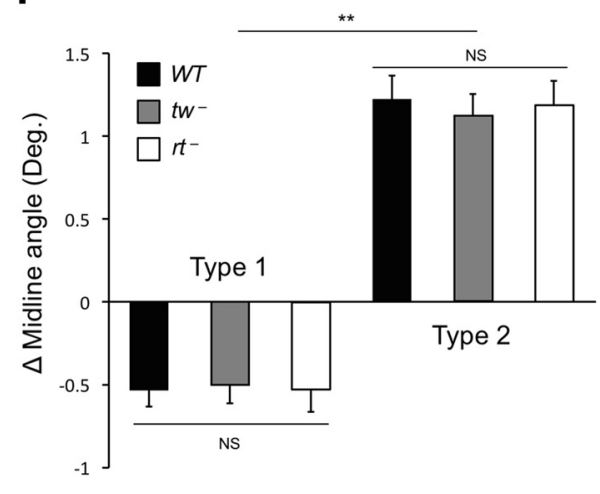

G

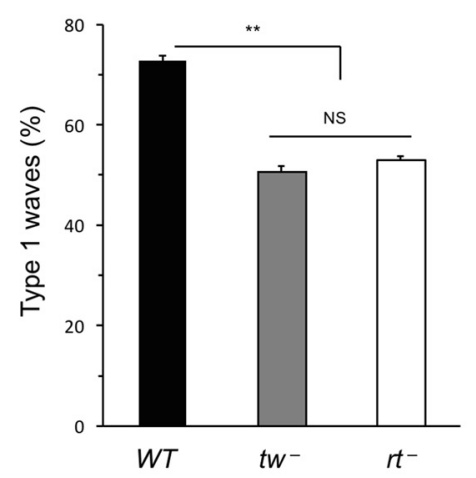

Figure 3. Body torsion phenotype of POMT mutants is associated with an abnormal pattern of contraction waves. $\boldsymbol{A}$, False-color images of type 1 and type 2 contractions in embryos expressing MHC-GFP (red pixels indicate higher signal). Scale bar, $100 \mu \mathrm{m}$. B, Profile examples of type 1 and type 2 waves. Contraction amplitude along embryo body is plotted over a period of one wave. Blue ovals indicate progression of contractions from posterior to anterior. Red oval indicates progression of type 2 wave from anterior to posterior during its first phase. Dashed red box indicates static contraction at the end of the first phase. Blue oval in type 2 wave corresponds to the second phase of the wave. $C$, Ratio between amplitudes of contraction in the end and the beginning of a wave calculated for type 1 forward and backward waves, and for the second phase of type 2 waves. $D$, Representative examples of contraction amplitude distribution along the embryo in type 1 forward wave (left) and type 2 wave (right). Contraction amplitude was measured for the same wave at posterior (A7, black line), middle (A4, red line), and anterior (T2, blue line) segments of the embry0. First and second phases of the type 2 wave are indicated. Contraction amplitude was analyzed using MHC-GFP. $\boldsymbol{E}$, Type 1 contractions have a similar effect on dorsal midline angle and cause right-handed body torsion regardless of genotype and direction of propagation. There is no statistical difference between any groups of data displayed. $\boldsymbol{F}$, Contractions of the same type yield statistically indistinguishable effects on torsion, regardless of genotype, while the effects of type 1 and type 2 waves within a given genotype are significantly different, being opposite in direction. G, POMT mutants generate smaller proportions of type 1 waves compared with wild-type embryos, though mutants are not significantly different from each other. Number of embryos and contractions analyzed: $\boldsymbol{C}, 10$ embryos for WT, 8 embryos for each mutant, and at least 20 individual contractions of each type for each genotype. $\boldsymbol{E}, 10$ embryos were analyzed for each $W T$ and $t w-$ genotypes, 8 embryos were analyzed for $r t^{-}$, and at least 20 contractions of each type were analyzed for each embryo. $\boldsymbol{F}, 10$ embryos for $W T$ and $t w$, at least 40 contractions of each type for each genotype; 8 embryos and at least 30 contractions of each type for $r t^{-}$. G, 10 embryos and $>130$ contractions for each genotype. $W T$, wild-type. $r t^{-}, r t^{p} / r t^{p}$. $t w^{-}, t w^{1} / t w^{1}$ (or $t w^{1} / Y$ ). In all panels, error bars indicate SEM. ${ }^{*} p<0.05,{ }^{* *} p<0.01$. NS, Not significantly different. Statistical analyses: $C, p>0.9$ for differences among data for type 1 waves, $p>0.7$ for type 2 waves, all genotypes; $p$ values for comparison between type 1 and type 2 waves for $W T, t w^{-}$, and $r t^{-}: 0.03,0.007$, and 0.008 , respectively. $\boldsymbol{E}, p>0.9$. $\boldsymbol{F}, p>0.9$ for differences among data for type 1 or type 2 waves, all genotypes; $p<1 \mathrm{E}-5$ for comparison between type 1 and type 2 waves. $\mathbf{G}, p<3 \mathrm{E}-5$ for differences between WT and $t w^{-}$or $r t^{-} ; p=0.86$ for $t w^{-} \mathrm{vs}^{-} t^{-}$comparison. $^{-}$ 


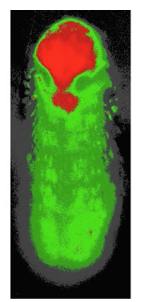

Movie 6. Example of type 1 contraction wave. Type 1 waves progress simply from one end of the embryo to the other. Contractions were false colored (most bright pixels are red) to help highlight the contraction wave. A forward wave in wild-type embryo is shown as an example. All tested genotypes generated type 1 waves similarly, although with different frequencies. Time series of images was recorded and sped up as in other movies.

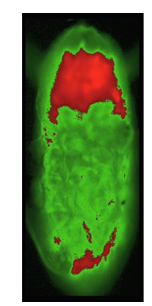

Movie 7. Example of type 2 contraction wave. Contractions were false colored to help visualize wave progression. The wave initiates near the anterior end, progresses toward the posterior, halts as static contraction, and is swept back to the anterior by a second wave phase. All tested genotypes generated type 2 waves similarly, but with different frequencies. Type 2 waves of opposite direction (i.e., originating at the posterior end) are practically absentinall analyzed genotypes exceptforsensmutants thatshow anoticeablenumberoftype initiated at the posterior end. Time series of frames was recorded and sped up as in other movies.

angle corresponding to the accumulation of left-handed torsion (Fig. $3 F$ ), which is consistent with the differential rolling mechanism and the fact that the amplitude of a type 2 wave increases on average during wave propagation (Fig. 3D). Our analysis indicated that type 1 waves constitute $\sim 73 \%$ of all waves in wildtype embryos on average, while the remaining $\sim 27 \%$ of coordinated contractions are type 2 waves. Mutant embryos, on the other hand, generate approximately equal numbers of type 1 and type 2 waves (Fig. $3 G$ ). These results suggest that type 2 waves normally serve as a compensatory mechanism to counteract the right-handed body torsion that would accumulate over the course of multiple type 1 waves. In POMT mutants, however, an abnormally high proportion of type 2 contractions results in the accumulation of a net left-handed torsion over time.

\section{Body torsion arises in mutants with PNS defects}

Abnormal coordination of muscle contractions and the failure to maintain body posture may be caused by defects in function of muscle and/or neurons, including PNS cells that were shown to be important for the generation of coordinated waves of muscle contractions (Suster and Bate, 2002; Hughes and Thomas, 2007; Song et al., 2007). To shed light on the cell-specific requirement of POMT activity for normal body posture, we applied a rescue strategy using UAS-GAL4-driven expression of a transgenic $t w$ construct in tw mutants. We found that a pan-neuronal driver (ELAV-Gal4) and a driver specific for class IV da sensory neurons (Ppk-Gal4) were each able to significantly rescue the embryo torsion phenotype. On the other hand, three different musclespecific drivers (DMEF2-GAL4, MHC-GAL4, and How-GAL4; Ranganayakulu et al., 1996; Schuster et al., 1996; Zaffran et al.,
1997) could not mediate the rescue of body torsion (Fig. 4A). These results suggested that $t w$ is required in neurons, and specifically in class IV da sensory neurons.

We hypothesized that POMTs function in the PNS to ensure a proper feedback from these cells to maintain normal body posture. This hypothesis predicts that PNS defects could result in an abnormal ratio of type 1:type 2 waves and cause torsion. To test this prediction, we examined embryos with significantly depleted PNS function caused by a defect in senseless (sens). Mutations in sens result in the loss of peripheral sensory neurons by stage 16 through cell-specific apoptosis (Nolo et al., 2000), thus impairing feedback from the PNS to the CNS in sens mutants. Although complete shutdown of sensory input halts peristaltic contractions entirely (Song et al., 2007), sens ${ }^{-}$embryos are still able to generate contraction waves, albeit with decreased frequency, presumably due to the fact that some da sensory neurons are still present in these embryos (Suster and Bate, 2002; Song et al., 2007). We analyzed sens ${ }^{-}$mutants and found that although they indeed generated fewer peristaltic contractions than wild-type embryos, individually these contractions were apparently normal type 1 or type 2 waves, with the same effects on rolling behavior and torsion as in wild-type embryos (Fig. $4 B, C$ ). However, different embryos generated type 1 and type 2 waves at highly variable ratios, with the proportion of type 1 waves ranging from $\sim 47 \%$ to $100 \%$ (Fig. 4D). These results are consistent with our hypothesis that sensory input can control the pattern of alternating type 1-type 2 contractions.

Furthermore, considering this variability of contraction patterns in sens mutants, our hypothesis predicts that some sens mutants should accumulate left-handed or right-handed torsion, depending on whether the proportion of type 1 waves they generate is substantially lower or higher than that observed in wildtype embryos ( $\sim 73 \%$ type 1 ; Figs. $3 G, 4 D)$, respectively. Indeed, when we analyzed the body posture of sens mutants, we found that $\sim 80 \%$ of embryos show body torsion, with left-handed and right-handed torsion defects being detected at approximately the same frequencies ( $\sim 40 \%$ each; Fig. $4 E, F)$. These data also supported our hypothesis that PNS cells participate in generating the proper pattern of alternating type 1-type 2 contractions, which is essential for the maintenance of body posture.

In further experiments, we analyzed $t w$-sens double mutants and revealed that $t w$ is epistatic to sens, as all double-mutant embryos were found to have left-handed torsion (Fig. $4 E$ ). Since da cells represent the only type of peripheral sensory neurons present in sens mutants (Nolo et al., 2000; Suster and Bate, 2002), this result suggests that the downregulation of POMTs in da neurons is sufficient to generate an abnormal feedback from these cells to the CNS and affect muscle contractions in $t w$-sens double mutants. However, these cells do not suffice to maintain proper contractions in sens mutants, presumably because normal contractions also require sensory feedback from other PNS cells that these mutants are missing (Suster and Bate, 2002; Hughes and Thomas, 2007). Together, these results suggest that PNS neurons, including class IV da neurons, while normally supporting the proper pattern of contraction waves, generate an aberrant feedback to the CNS in POMT mutants. This abnormal feedback affects the pattern of muscle contractions and thus underlies the pathogenic mechanism of the torsion phenotype.

\section{POMTs are required for patterning of axonal projections of sensory neurons}

To elucidate possible cellular mechanisms underlying the requirement of POMTs for PNS function, we examined POMT mutants for 
A

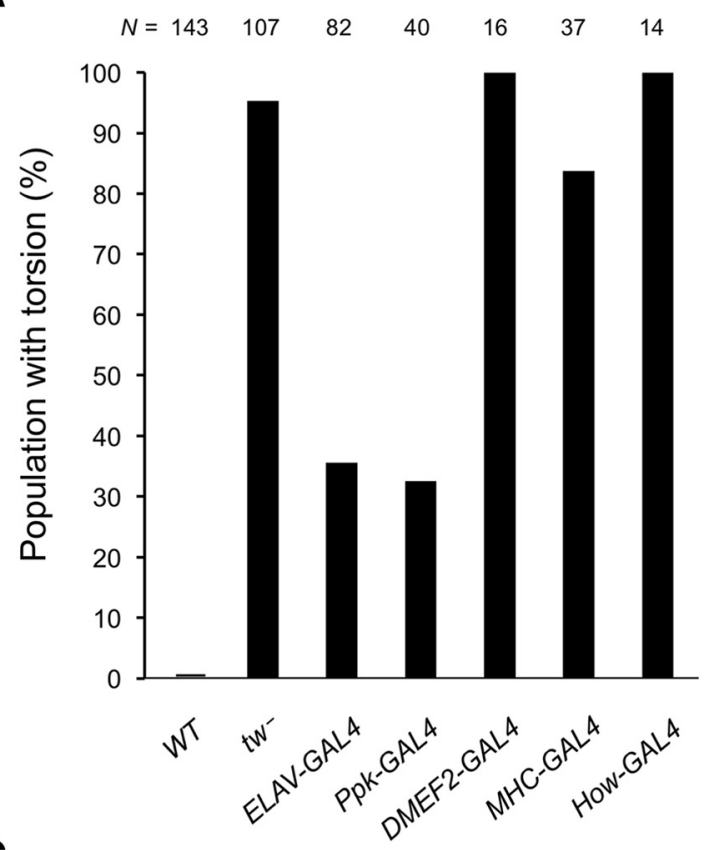

C

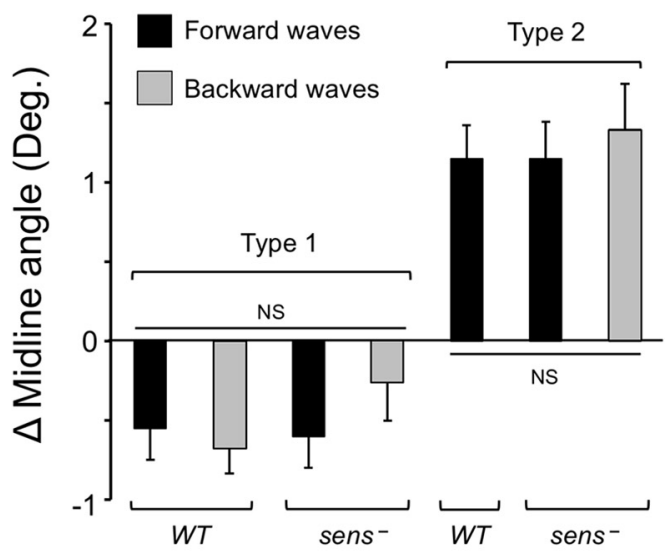

$\mathbf{E}$

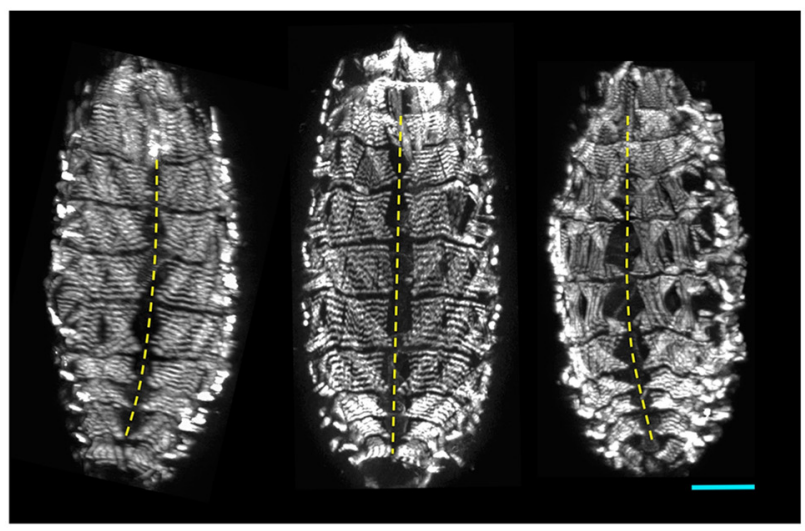

RH Torsion

No Torsion

LH Torsion
B

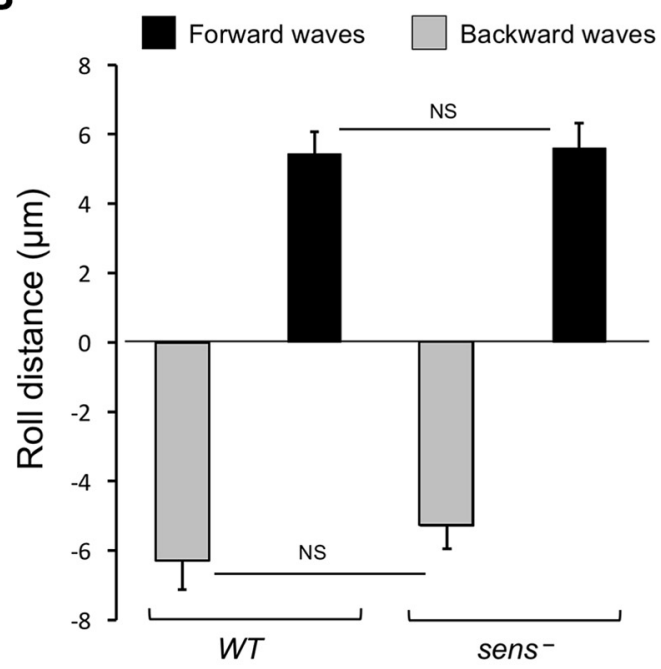

D

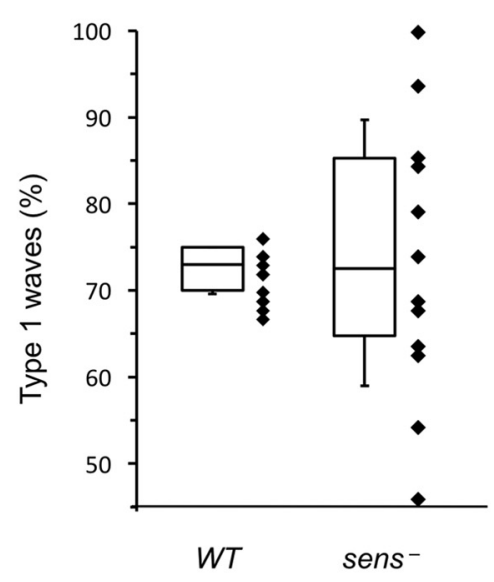

F

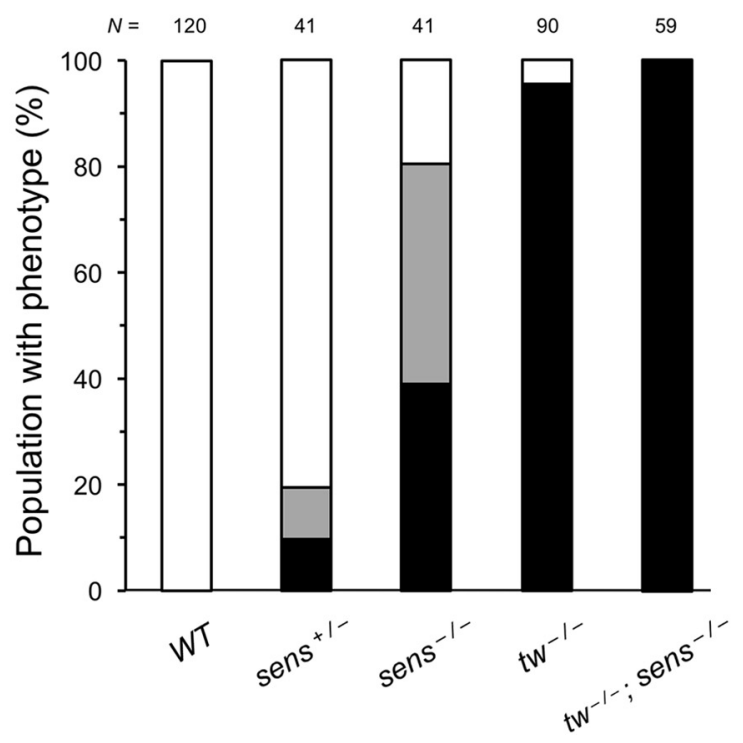

LH Torsion

RH Torsion

No Torsion

Figure 4. POMTs are required in peripheral sensory neurons for the maintenance of posture. $A$, Rescue of embryonic torsion by transgenic expression of UAS-tw construct in tw mutants using cell-specific drivers. Expression in all neurons (ELAV-GAL4) or in class IV da neurons (Ppk-GAL4) can substantially rescue torsion, while muscle-specific expression using DMEF2-GAL4, MHC-GAL4, and How-GAL4 cannot rescue the torsion phenotype (the presence of a driver or the UAS-tw transgene alone did not affect the torsion phenotype, which was confirmed in control experiments). Genotypes are designated as follows: WT, wild-type. $t w^{-}, t w^{7} / t w^{7}$ (or $t w^{1} / M$ ). ELAV-GAL4, $t w^{7} / t w^{7}\left(\right.$ or $t w^{7} / M$; UAS-tw/ELAV-GAL4. Ppk-GAL4, $t w^{7} / t w^{7}\left(\right.$ or $\left.t w^{1} / M\right)$; UAS-tw/+; (Figure legend continues.) 
possible defects of PNS functional morphology. Since our rescue and genetic interaction experiments suggested that the function of POMTs could be required in class IV da sensory neurons, we focused on the analysis of these cells in POMT mutants. Class IV da cells develop a characteristic laminar pattern of axonal projections in the ventral ganglion (Grueber et al., 2007). We labeled these projections using a $P p k$-tdTom reporter or a Ppk-Gal4driven UAS-tdGFP marker (Han et al., 2012) and analyzed their morphology once embryos hatched from the shell (the first instar stage). We found that the axonal projections show significant abnormalities in $t w$ and $r t$ mutants, including thickened commissural branches and, in the case of $r t$ mutants, missing or severely depleted longitudinal tracts (Fig. 5A-E). The axon phenotype of tw mutants was milder on average, which is consistent with the fact that the $t w^{1}$ mutant used in these experiments is a hypomorph (Lyalin et al., 2006). Transgenic expression of $t w$ or $r t$ induced specifically in class IV da cells could rescue defects of axonal projections in $t w$ or $r t$ mutants, respectively (Fig. $5 B-E$ ), which confirmed the specificity of the phenotypes and also suggested that POMTs function cell autonomously to pattern axonal projections of sensory neurons. Furthermore, the rescue of axonal projections correlated with the rescue of muscle contractions in $t w$ mutants, supporting the hypothesis that the miswiring of sensory axons causes the defect of muscle contractions in POMT mutants (Fig. 5F).

To characterize the nervous system phenotype in more detail, we used markers for glial cells and major axonal tracts in the ventral ganglion. Immunostaining with Repo antibody that specifically labels the majority of glial cells (Dickson and Zou, 2010; NeuhausFollini and Bashaw, 2015) revealed no significant difference between wild-type and $r t$ mutants (Fig. $6 A, B$ ), which suggests that glia development is not affected in POMT mutants. This result, however, cannot rule out the possibility of some functional defects of glial cells, in particular, possible abnormalities in midline glia that plays role in axon guidance (Jacobs, 2000; Crews, 2010). To check for possible abnormalities in axon guidance, we examined immunostaining for FasII that labels all major long axon tracts in the ventral ganglion (Landgraf et al., 2003; Wu et al., 2011). These tracts are affected by mutations in genes involved in major axon guidance pathways (e.g., defects in the Slit/Robo pathway cause abnormal midline crossing;

$\leftarrow$

(Figurelegend continued.) Ppk-GAL4/+.DMEF2-GAL4, $\mathrm{tw}^{1} / \mathrm{tw}^{1}\left(\right.$ or $t w^{1} /$ ); UAS-tw/+;DMEF2GAL4/+. MHC-GAL4, $t w^{1} / t w^{1}$ (or $\left.t w^{1} / Y\right)$; UAS-tw/+; MHC-GAL4/+. How-GAL4, $t w^{1} / t w^{1}$ (or $t w^{1} / M$; UAS-tw/How-GAL4. B, Effect of forward and backward type 1 contractions on rolling in sens mutants compared with wild type. There is no significant difference between wild-type and sens mutants for corresponding waves ( $p=0.98$ ). C, Effect of type 1 and type 2 contractions on torsion in sens mutants. As in other genotypes, type 1 and type 2 waves result in negative (right-handed torsion) and positive (left-handed torsion) midline angles, respectively. In sens mutants, unlike any other genotype analyzed, a significant number of type 2 backward contractions were observed. Similar to type 2 forward waves, these also resulted in left-handed torsion. There were no significant differences between groups of data within a given contraction type $(p>0.05)$. D, Although WT and sens ${ }^{-}$embryos generate a similar proportion of type 1 waves on average, sens mutants have a much greater variability in the proportion of type 1 waves between individual embryos (black diamonds), suggesting that some embryos should develop body torsion. $E$, Examples of sens mutants with left-handed (LH) or right-handed (RH) body torsion, or without body torsion phenotype. $F$, Proportions of embryos with left-handed torsion, right-handed torsion, and no detectable torsion among WT, sens ${ }^{-},{ }^{-} w^{-}$, and $t w^{-}$; sens ${ }^{-}$ double mutants. $N$, Number of embryos analyzed for each genotype. All panels: $W T$, wild-type. Sens ${ }^{-}$, sens ${ }^{E 2} . T w^{-}, t w^{1} \cdot t w^{-1-}$; sens ${ }^{-1-}, t w^{1} / t w^{1}\left(\right.$ or $\left.^{1} w^{1} / Y\right)$; sens ${ }^{E 2} /$ sens $^{E 2}$. Corresponding wild-type alleles are indicated by " + ." Unless indicated otherwise, at least 12 embryos and 15 contractions per embryo were assessed for each genotype. Statistical analyses: $C, p>0.9$ for differences among data for same contraction type, $p<1 \mathrm{E}-5$ for differences between type 1 and type 2 contractions, all genotypes. $\boldsymbol{D}, p=0.97$
Jacobs, 2000), Plexin and Semaphorin mutants show disorganized morphology of selected tracts (Ayoob et al., 2006; Wu et al., 2011), while mutations in Netrins and frazzled affect tract continuity (Organisti et al., 2015). We found no obvious abnormalities of long axon tracts in $r t$ mutants (Fig. $6 C-F$ ). The FasII-positive tracts also provided a useful reference for examining the dorsoventral position of sensory projections in the ventral ganglion (Grueber et al., 2007). Analysis of optical sections stained for FasII revealed that class IV sensory axons project properly to the ventral region of the ganglion in $r t$ mutants (Fig. 6G,H). Together, these results suggest that axon guidance is not generally affected by POMT mutations.

We further investigated in greater detail the phenotype of sensory projections by labeling individual class IV axons using the Flp-out technique (Basler and Struhl, 1994; Wong et al., 2002; Grueber et al., 2007). These experiments revealed that the morphology of sensory axon termini in $r t$ mutants is different from that in wild-type controls. In agreement with a previous report (Grueber et al., 2007), we found that individual axon termini in wild-type ventral nerve cords fall into the following two distinct categories: the axons that branch along longitudinal tracts of the laminar pattern without crossing the midline ("branching only"); and the axons that both branch longitudinally and cross the midline ("branching and crossing"). In $r t$ mutants, in addition to these two types of axons, we observed a substantial proportion of axons that cross the midline without branching ("crossing only"; Fig. 6I,J). This type of axon was not present in wild-type ventral nerve cords. The crossing-only axons appear to develop at the expense of branching and crossing axons, as the proportion of branching-only axons was about the same in mutants and controls $(20 \%$ and $19 \%$ in $r t$ mutants and wild-type, respectively; Fig. $6 J$ ). These data are consistent with the phenotype of the laminar pattern of sensory axon projections in POMT mutants that shows thickening of commissural tracts and depletion of longitudinal connections (Fig. 5A,B). However, other possible abnormalities of axon termini, such as defects in fasciculation or branch distribution, may also contribute to the phenotype of the laminar pattern, but they could not be revealed in our Flp-out experiments. Further studies using multiple markers will be required for more detailed analysis of individual sensory axon termini in POMT mutants. Nevertheless, together, our results strongly support the hypothesis that POMTs are specifically required for proper patterning of class IV sensory axon termini, but protein O-mannosylation is not involved in general axon guidance or overall development of the nervous system.

\section{Dystroglycan mutants do not have the torsion phenotype}

$\mathrm{Dg}$ is the best characterized target of protein O-mannosylation. POMT-mediated glycosylation of Dg is important for its functional interactions with ECM ligands (Barresi and Campbell, 2006; Yoshida-Moriguchi and Campbell, 2015). While O-mannosylation was found on many other mammalian glycoproteins (Vester-Christensen et al., 2013; Panin and Wells, 2014), Dg is so far the only known O-mannosylated protein in Drosophila (Nakamura et al., 2010a,b). To test whether the torsion phenotype of POMT mutants is caused by a defect in Dg activity, we analyzed first instar $D g$ mutant larvae. While nearly all first instar POMT mutant larvae showed conspicuous body torsion (Fig. 1B), no torsion phenotype was detected in $D g$ mutants (Fig. 7). These results suggest that the torsion phenotype of POMT mutants is not caused by the loss of Dg activity and that some other yet unknown functional substrates of POMTs play a role in the pathogenic mechanisms underlying this phenotype. 
A

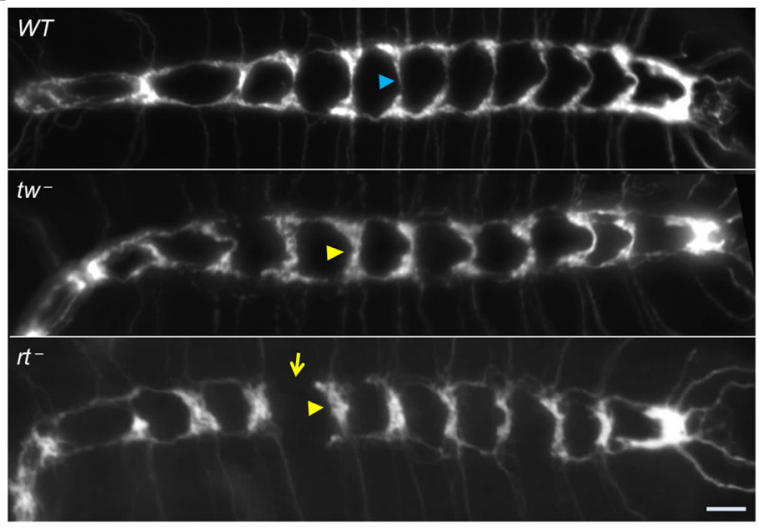

B

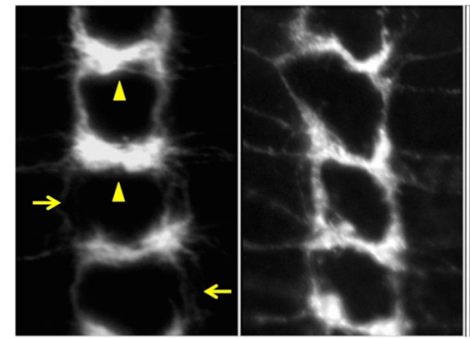

$r t-$

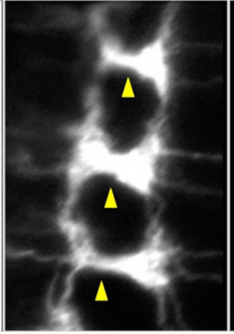

$t w^{-}$

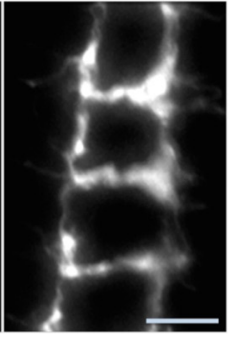

$t w-p p k>t w$
C

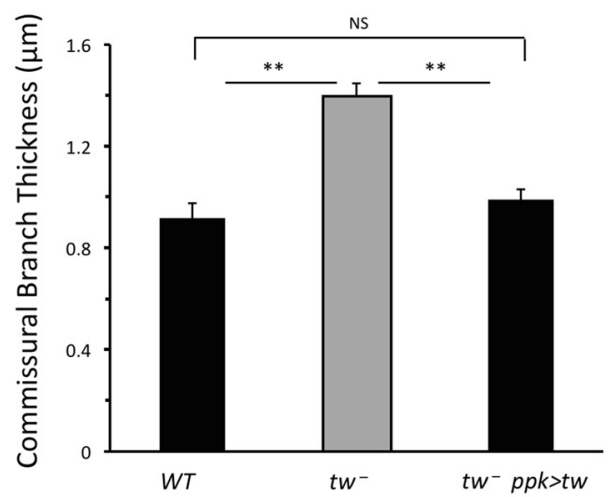

E

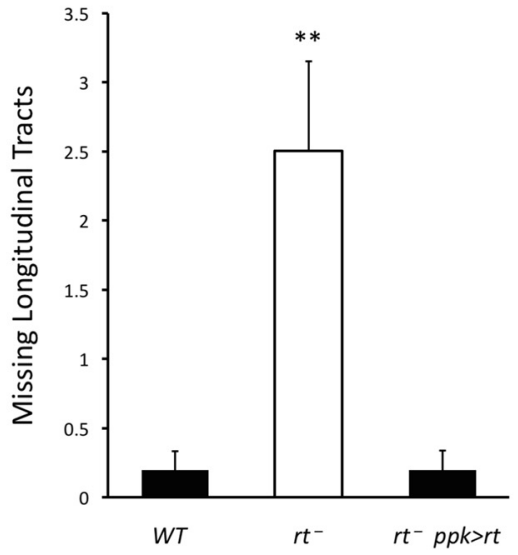

D

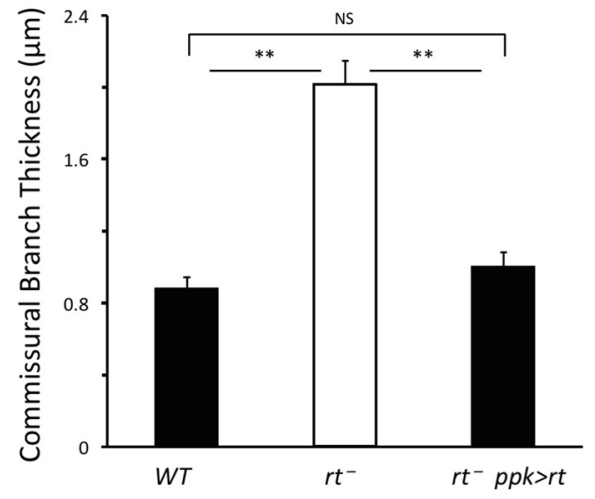

$\mathbf{F}$

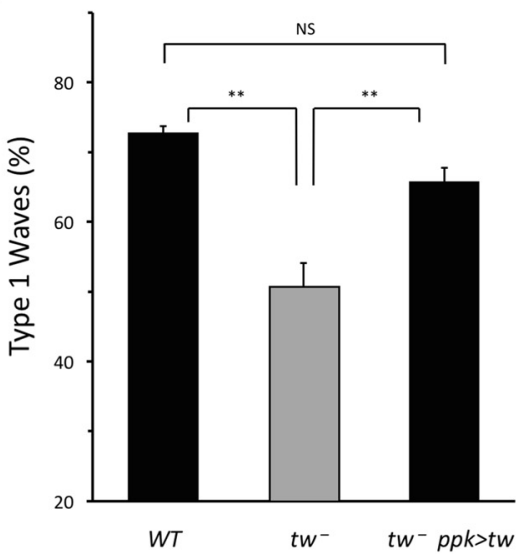

Figure 5. POMTs affect the function of peripheral sensory neurons. A, POMT mutants have abnormal axonal projections of class IV da sensory neurons in the ventral ganglion. The laminar pattern of axonal projections shows abnormally thickened commissural tracts in $r t$ and tw mutants (yellow arrowheads, compared with tracts indicated by blue arrowheads in WT control), and missing longitudinal tract in $r$ t mutants (arrow). $\boldsymbol{B}-\boldsymbol{F}$, Transgenic expression of POMTs in class IV da neurons can rescue the phenotype of axonal projections $(\boldsymbol{B}-\boldsymbol{E}$ ) and abnormal pattern of contraction waves $(\boldsymbol{F}) . \boldsymbol{B}$, Representative images of the same region of the laminar pattern (neuromeres a1 -3 that are more frequently affected in POMT mutants) are shown for mutant and rescue genotypes. The phenotypes of thickened commissural branches (arrowheads) and depleted longitudinal tracts (arrows) are rescued by transgenic expression of POMTs in class IV da neurons. Number of animals analyzed for each genotype: WT, 14; $t w^{-}, 15 ; t w^{-}, p p k>t w$ (rescue), $14(\mathbf{C}) ; W T, 10 ; r t^{-}, 10 ; r t^{-}, p p k>r t$ (rescue), 11. (D-E). Number of contractions (embryos) analyzed in $\boldsymbol{F}: W T, 186$ (10); $t w^{-}, 163(10) ; t w^{-}$, $p p k>t w$ (rescue), 128 (8). In all panels, errorbars indicateSEM. ${ }^{* *} p<0.01$; ns, not significantly different. WT, wild-type. $t w^{-}, t w^{1} / t w^{1}\left(\right.$ or $\left.t w^{1} / M\right) . t w^{-} ; p p k>t w, t w^{1} / t w^{7}\left(\right.$ or $\left.t w^{1} / M\right) ; P p k-G A L 4 /+; U A S-t w /+$. $r t^{-}, r t^{2} / r^{2} . r t^{-}, p p k>r t, r t^{2} P p k-G A L 4 / r^{2}$ UAS-rt. Driver-only and UAS-transgene-only control genotypes were also analyzed for corresponding rt and tw mutant alleles and found to be indistinguishable from mutants alone. Axonal projections were visuaized with Ppk-tdGFP (Han et al., 2012). Anterior is to the left $(\boldsymbol{A})$ or up (B). Scale bar, $10 \mu \mathrm{m}$. Statistical analyses: $(\boldsymbol{C}-\boldsymbol{F}$, $p$ values for differences between $W T$ and mutant, rescue and mutant, and between WT and rescue genotypes, respectively: 4E-5, 3E-4, 0.79 (C);6E-9, 4E-8, 0.62 (D); 1E-3, 8E-4, 0.98 (E); 1E-6, 4E-4, 0.18 (F).

\section{Discussion}

Here we described a novel embryonic phenotype in POMT mutants and investigated its mechanism using genetic tools and liveimaging approaches. We focused on a newly discovered role of
POMTs in generating patterns of muscle contractions. Our detailed analyses of muscle contractions led us to the main conclusion that the torsion phenotype arises due to abnormal coordination of contraction waves. We found that during late embryogenesis embryo 

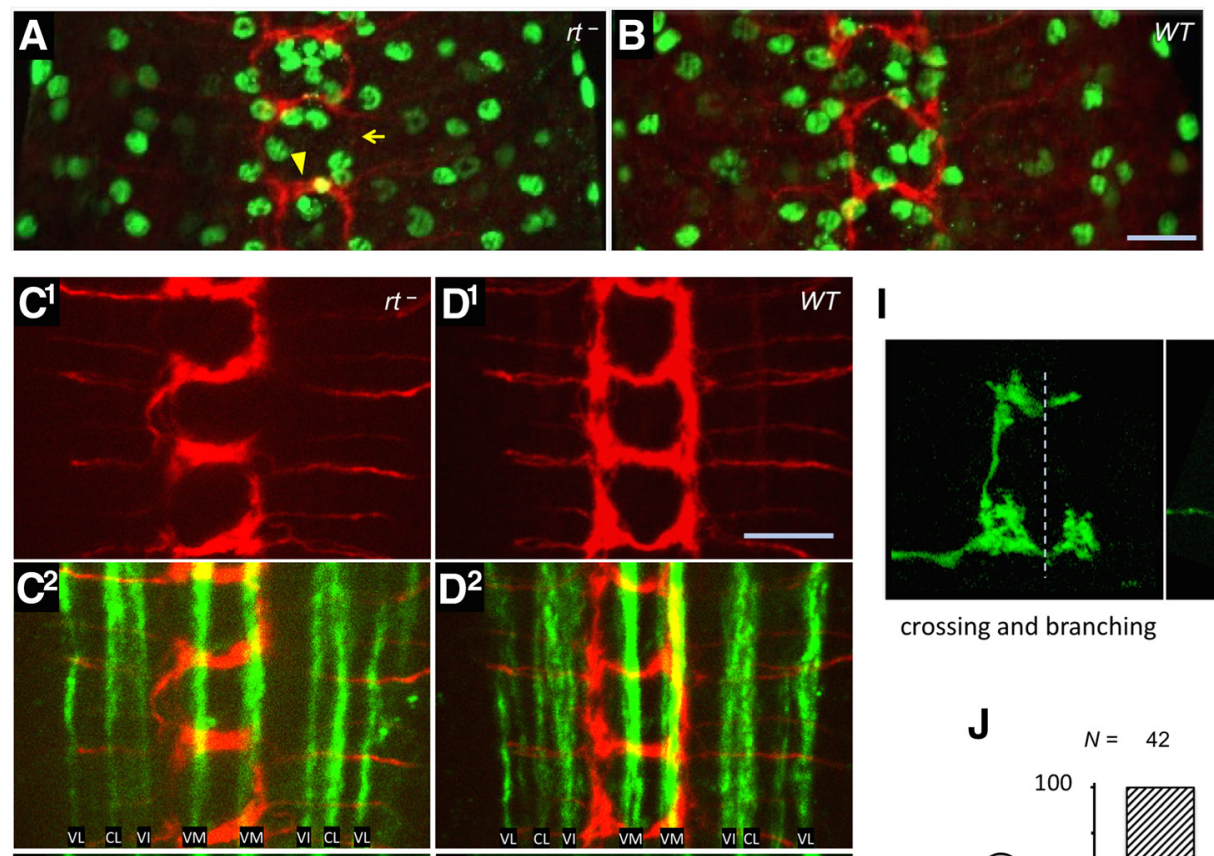

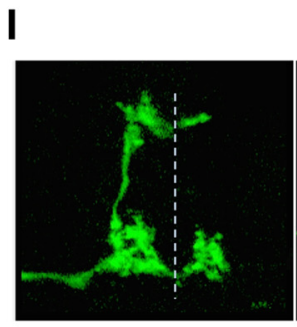

crossing and branching

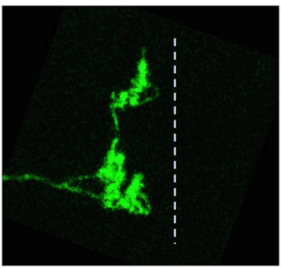

branching only

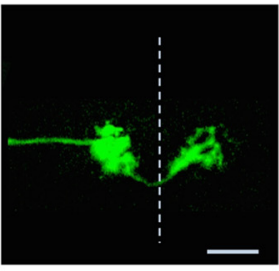

crossing only

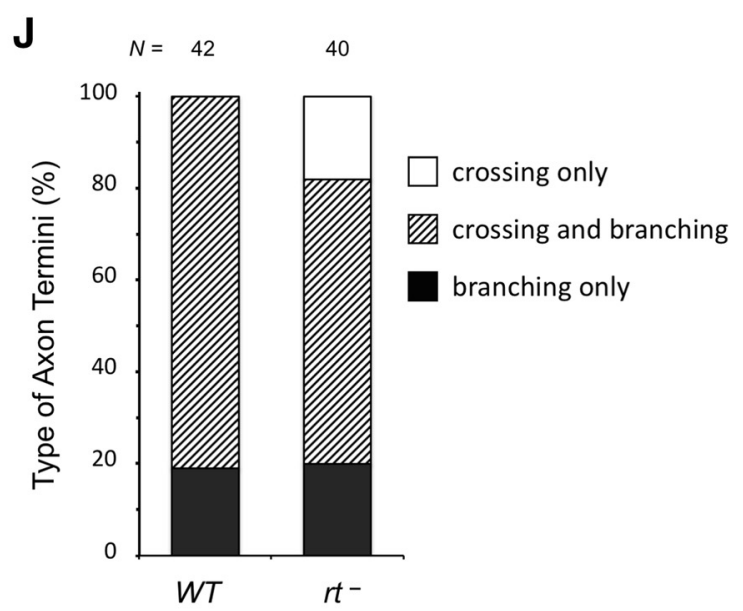

Figure 6. POMT mutations do not generally affect glia development and axon pathfinding, while being associated with defects in patterning of sensory axon termini. $\boldsymbol{A}, \boldsymbol{B}$, Immunostaining with anti-Repo antibody that specifically labels glial cells (green) revealed no significant defects in the distribution and number of glial cells in $r t$ mutants (22.1 \pm 0.9 and $23.7 \pm 0.8$ glial cells per

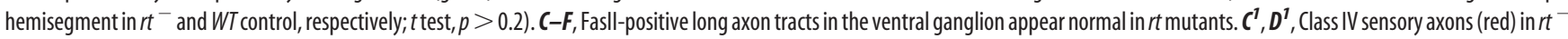
and wild-type control, respectively. $\boldsymbol{C}^{\mathbf{2}}, \boldsymbol{D}^{\mathbf{2}}$, overlay of the axon staining shown above $\left(\boldsymbol{C}^{\mathbf{1}}, \boldsymbol{D}^{\mathbf{1}}\right)$ with the staining of long axonal tracts (Fasll, green) in the ventral part of the ganglion. The same region of the ventral ganglion corresponding to neuromeres a1-3 is shown for both genotypes. $\boldsymbol{E}, \boldsymbol{F}$, Fasll immunostaining of long axonal tracts in dorsal part of the ganglion in $r t^{-}$and wild-type, respectively. $\mathbf{G}, \boldsymbol{H}$, Z-projections reconstructed from stacks of horizontal optical sections through the ventral ganglion show similar dorsoventral lamination of class IV axon termini (red) in $r t^{-}$and wild-type, respectively, based on their location relative to Fas II tracts (green). The commissural branch of neuromere a 2 is shown for both genotypes. $I$, Examples of individual class IV sensory axons with "crossing and branching," "branching only," and "crossing only" types of morphology, relative to the midline (dashed line). J, Quantification of types of class IV sensory axons revealed by Flp-out clones in wild-type and $r t$ mutants. N, Number of individual axons analyzed. Nomenclature of Fasll tracts is according to the study by Landgraf et al. (2003): C, central; D, dorsal; I, intermediate; L, lateral; M, median; V, ventral. Axons were visualized using Ppk-tdTom (A-H; Han et al., 2012) or Flp-out technique (I, J; see Materials and Methods). Images in $\boldsymbol{C}-\boldsymbol{F}$ represent projections of stacks of optical sections through ventral and dorsal regions of the ventral ganglion, respectively. $r t^{-}, r t^{2} / r t^{2}$. Scale bar, $10 \mu \mathrm{m}$.

body posture is controlled dynamically by contraction waves. During this time, left-handed torsion gradually accumulates in POMT mutants. The proper pattern of contraction waves presumably requires a PNS-mediated feedback that relays information on body posture to the CNS. The pattern of waves depends on POMT activity in the nervous system, including class IV sensory da neurons. In POMT mutants, sensory neurons apparently generate an aberrant signal to the CNS, which results in an abnormal pattern of contraction waves and the torsion phenotype (Fig. 8).

Several lines of evidence provided support for this mechanism. First, our data suggest that the torsion phenotype is not caused by gross defects of muscle morphology (Fig. 1A). Although we cannot completely exclude the possibility that muscles may have some fine morphological defects or/and abnormal physiology, our analyses suggested that embryonic muscles, rather than being misaligned during development, are formed properly in POMT mutants. The torsion phenotype arises only during peristaltic contractions and is essentially fully penetrant (Figs. 1,4). Occasional muscle defects were reported in POMT mutants, but these defects are present at low frequency and are not bilaterally biased (MartínBlanco and García-Bellido, 1996; Haines et al., 2007; Ueyama et al., 2010), and thus it was previously concluded that they are unlikely to cause the misalignment of body segments (MartínBlanco and García-Bellido, 1996). This is consistent with our results, which furthermore indicated that the torsion phenotype is developmentally transient, as the phenotype becomes undetectable at later larval stages (Fig. $1 B$ ). This presumably happens because the torsion becomes fixed as the cuticle hardens during late embryogenesis, but the phenotype disappears once the cuticle is shed upon transition to the second instar. Second, the torsion arises due to alteration in the pattern of contraction waves, 
while the effects of individual waves on posture are not altered in POMT mutants (Figs. $2 B-D, 3 C-G$ ). These data suggest that individual muscles contract normally and that the torsion is caused primarily by a neurological abnormality affecting wave patterning. Third, our analyses of sens mutants highlighted the role of PNS cells in producing the torsion phenotype. A defect in the specification of PNS neurons alone could cause muscle contraction and posture phenotypes similar to those in POMT mutants (Fig. 4D-F). Epistatic interaction between $t w$ and sens suggested that da sensory neurons are sufficient to propagate the abnormal sensory feedback in POMT mutants (Fig. $4 F$ ). Furthermore, $P O M T$ mutations specifically affect patterning of axon termini, while axon guidance and the ventral ganglion development are not generally affected (Figs. 5, 6), suggesting that aberrant connectivity between sensory and CNS neurons underlies the defect of sensory feedback. Finally, genetic rescue revealed that POMT activity restored in class IV da sensory neurons in POMT mutants was sufficient to rescue the torsion phenotype and both the abnormality of contractions and the defect in axonal projections (Fig. $5 B-F$ ), suggesting that these neurons play an important role in POMT-mediated control of contractions and body posture. This role is consistent with the proposed function of these polymodal neurons in proprioception and mechanosensation and their involvement in the regulation of larval locomotion (Adams et al., 1998; Ainsley et al., 2003; Zhong et al., 2010; Gorczyca et al., 2014).

We found that $D g$ mutants do not have body torsion, suggesting that the torsion phenotype of POMT mutants is not caused by the loss of Dg activity and other POMT target(s) could affect sensory axon patterning. Two main orthogonal systems that position sensory axons along dorsoventral and mediolateral axes rely on Semaphorin/Plexin and Slit/Robo signaling, respectively, while connectivity across the midline also depends on the Netrin/Frazzled pathway (Zlatic et al., 2003, 2009; Grueber et al., 2007; Morikawa et al., 2011). POMT mutants have sensory axons properly positioned along the dorsoventral axis, suggesting that POMTs may affect primarily mediolateral and/or midline-crossing cues. However, axon guidance is not generally abnormal in POMT mutants, and defects are probably limited to the patterning of axon termini. Proper patterning of axon termini is important for correct circuit assembly; however, this mechanism remains poorly understood and probably involves the cooperation of canonical cues with additional modulators (Kolodkin and Tessier-Lavigne, 2011), such as cadherins, RPTPs, and other adhesion and signaling molecules (Morikawa et al., 2011; Dascenco et al., 2015; Organisti et al., 2015). Whether some of these proteins can be relevant POMT targets remains to be elucidated.

Our experiments discovered an intrinsic chirality of embryo rolling behavior during peristaltic waves of muscle contractions. Although the mechanism underlying this behavior remains unknown, considering that Drosophila body wall muscles show no obvious asymmetry in development, a putative handedness of
WT $D g^{-}$
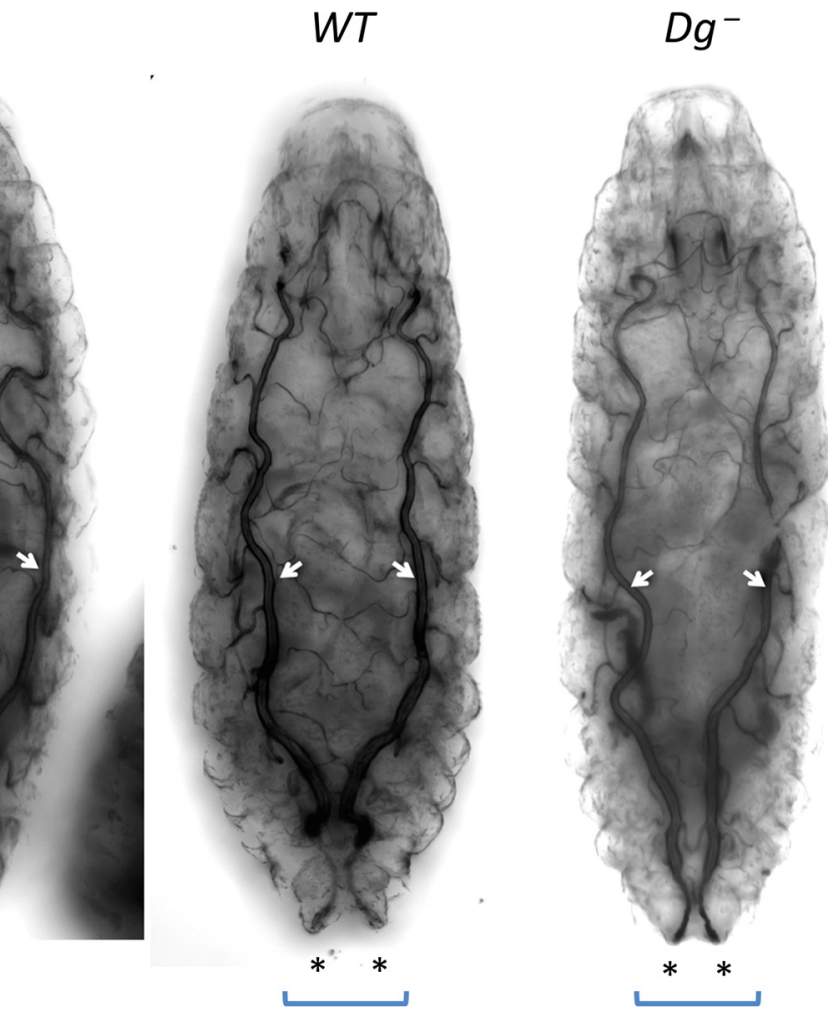

Figure 7. Genetic inactivation of $D g$ does not result in body posture abnormality. While tw mutants show conspicuous body posterior spiracles (asterisks), Dg mutants show no torsion and are indistinguishable from wild-type control (WT). Representative images of fixed first instar larvae obtained with transmitted light illumination. $D g^{-}, D g^{086} / D g^{086} ; t w^{-}, t w^{7} / t w^{7}\left(o r t w^{7} / Y\right) ; W T$, wild-type control with matching genetic background (Canton-S). More than 20 larvae were analyzed for each genotype.

embryo-eggshell interactions may provide an explanation of this phenomenon. Irrespective of the exact source of the chirality, here we propose a model that explains the relationship between rolling and torsion based on the assumption that rolling forces correlate with the strength of contractions. We propose that chiral rolling forces are induced by the propagation of a contraction wave, perhaps through interactions between cuticle and the vitelline membrane, and result in left-handed embryo rolling with respect to wave propagation. This model is supported by the observation that both backward and forward type 1 waves result in counterclockwise embryo rolling, when viewed in the direction of wave propagation. Our model predicts that these waves induce stronger rolling in the beginning and weaker rolling in the end of propagation since they show on average greater amplitude in the beginning and decreasing amplitude toward the end of the wave (Fig. $3 C, D$ ). This should generate a differential distribution of a torque force along the embryo, and, therefore, embryo torsion. Importantly, while waves in the opposite direction generate rolling in different directions, this torque force is predicted to induce right-handed (dextral) body torsion, regardless of wave direction (Fig. 8A), which is supported by our data (Fig. $3 E, F$ ). Our model is also consistent with the fact that biphasic (type 2) waves generate a left-handed (sinistral) torsion. According to our observations, type 2 waves induce differential rolling mostly at the anterior part, during the end of the wave, presumably because this part experiences stronger contractions (and thus greater rolling) in the second phase of the wave, compared with relatively weaker contractions (and therefore less rolling) in the initial phase that induces rolling in the opposite direction (Fig. 8). Rolling is essentially halted at the posterior end, where the backward 
A

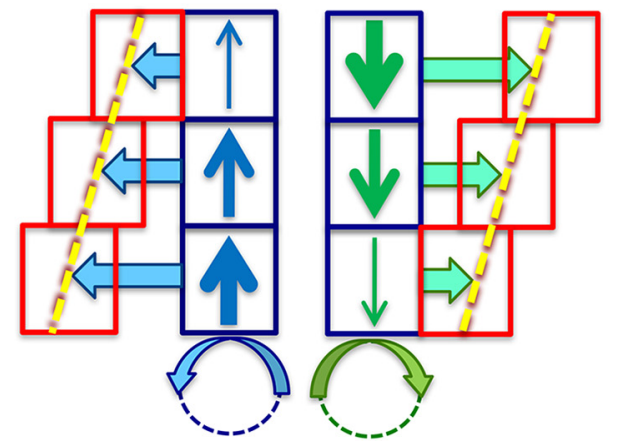

Type 1 Waves

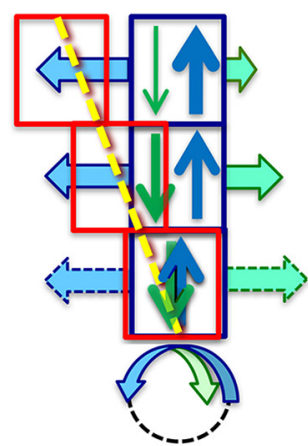

Type 2 Waves
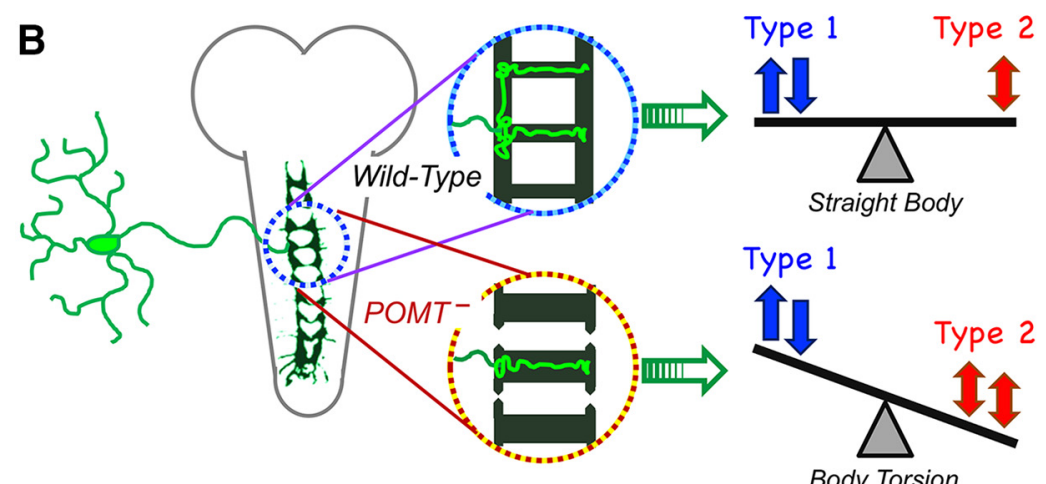

Body Torsion

\section{Sensory Neuron CNS Sensory Axon Termini Muscle Contractions}

Figure 8. Model of the dynamic control of body posture by peristaltic waves of muscle contractions in Drosophila embryos. $A$, Effect of contraction waves on body torsion. Type 1 and type 2 waves have opposite effects on posture, inducing right-handed and left-handed torsion, respectively. These effects result from differential embryo rolling within the eggshell. Upon wave propagation, embryos roll in a chiral manner, while displacement of individual segments depends on the strength of muscle contractions. Type 1 waves induce right-handed torsion because these contractions are on average stronger at their point of initiation (solid vertical blue and green arrows of different thickness represent propagating contractions of varied strength). This causes an accumulation of torsion over multiple waves, regardless of direction of wave propagation (empty horizontal blue and green arrows represent distance rolled, red boxes show overall segment displacement). Type 2 waves include two phases that correspond to contractions propagating in opposite directions. Type 2 waves induce left-handed torsion because first-phase and second-phase contractions cancel the effect of each other in the posterior but not the anterior part of the embryo, where the second phase induces stronger rolling. $\boldsymbol{B}$, Control of body posture by sensory feedback. To maintain straight posture, sensory neurons send feedback to the CNS to control the pattern of alternating type 1-type 2 waves. In POMT mutants, sensory axon termini are abnormal, which affects sensory circuit assembly, causing aberrant feedback from sensory neurons. This compromises the control of the wave pattern and results in an excessive proportion of type 2 waves, which leads to body torsion.

and forward waves overlap and cancel the effect of each other, inducing prolonged static contractions (Fig. 3A,B,D; Movie 7). As a result, only the anterior part of the embryo experiences a significant net roll, producing a cumulative left-handed torsion, which is in agreement with observations (Fig. $3 F$ ).

Interestingly, POMT mutations affect body posture by changing the pattern of waves, while rolling and torque associated with individual waves remain unaffected. Therefore, POMT mutations do not break the symmetry of processes that control posture. Instead, they uncover the intrinsic chirality underlying these processes by impairing the nervous system-mediated mechanism that compensates for this chirality and maintains the alignment of body segments. The dynamic correction for chiral torsion that we described here provides, to our knowledge, the first example of a "chirality-neutralizing" mechanism that maintains the symmetry of a developing system affected by asymmetric forces.

Here we identified a new asymmetric marker in Drosophila development, an embryo rolling behavior. How is this chiral- ity related to other known markers of LR asymmetry? While LR asymmetry affects numerous aspects of the development and physiology of more complex animals, LR asymmetry in Drosophila is relatively simple. It appears to be limited to the morphogenesis of some tubular structures, including male terminalia rotation and looping of the gut and testis (Géminard et al., 2014). These LR asymmetries are thought to depend on cellular interactions and common molecular regulators such as Myosin ID (Géminard et al., 2014). It is tempting to speculate that the rolling asymmetry may be "hardwired" in intrinsic molecular chirality of some structural features of cuticle, eggshell, or muscles, and thus the rolling asymmetry and other LR markers may have distinct mechanisms. Further studies are required to reveal the relationship between embryo asymmetric rolling and other LR markers.

The striking similarity of embryo torsion and adult abdomen rotation phenotypes of POMT mutants suggests that they result from analogous pathogenic mechanisms. However, body torsion disappears upon transition from the first to second instar, indicating that the abdomen rotation phenotype is induced de novo during metamorphosis. Intriguingly, coordinated waves of abdominal muscle contractions that facilitate prepupa-pupa transition occur during metamorphosis (Robertson, 1936; Chadfield and Sparrow, 1985; Fortier et al., 2003). Putative abnormalities of these contractions may cause misalignment of abdominal segments, resulting in the abdomen rotation phenotype. Further experiments will be needed to test this hypothesis.

Remarkable conservation of POMTs in animals suggests an intriguing possibility that their role in facilitating neural connectivity to control muscle contractions may be conserved in vertebrates. Interestingly, the downregulation of POMK, a kinase involved in biosynthesis of vertebrate O-mannosyl glycans, causes abnormality in coordinated muscle contractions in zebrafish embryos, while clinical features of syndromes associated with POM defects commonly include abnormal muscle contractions (Leyten et al., 1996; Di Costanzo et al., 2014). Further studies should shed light on evolutionary conservation of the role of POMTs in regulating muscle contractions and axonal connectivity, which can potentially elucidate pathogenic mechanisms of diseases caused by POM abnormalities.

\section{References}

Adams CM, Anderson MG, Motto DG, Price MP, Johnson WA, Welsh M] (1998) Ripped pocket and pickpocket, novel Drosophila DEG/ENaC subunits expressed in early development and in mechanosensory neurons. J Cell Biol 140:143-152. CrossRef Medline

Ainsley JA, Pettus JM, Bosenko D, Gerstein CE, Zinkevich N, Anderson MG, Adams CM, Welsh MJ, Johnson WA (2003) Enhanced locomotion 
caused by loss of the Drosophila DEG/ENaC protein Pickpocket1. Curr Biol 13:1557-1563. CrossRef Medline

Ayoob JC, Terman JR, Kolodkin AL (2006) Drosophila Plexin B is a Sema-2a receptor required for axon guidance. Development 133:21252135. CrossRef Medline

Barresi R, Campbell KP (2006) Dystroglycan: from biosynthesis to pathogenesis of human disease. J Cell Sci 119:199-207. CrossRef Medline

Basler K, Struhl G (1994) Compartment boundaries and the control of Drosophila limb pattern by hedgehog protein. Nature 368:208-214. CrossRef Medline

Bridges CB, Morgan TH (1923) The third-chromosome group of mutant characters of Drosophila melanogaster. Carnegie Institute of Washington Publication 327:1-251.

Chadfield CG, Sparrow JC (1985) Pupation in Drosophila melanogaster and the effect of the Lethalcryptocephal mutation. Dev Genet 5:103-114.

Christoforou CP, Greer CE, Challoner BR, Charizanos D, Ray RP (2008) The detached locus encodes Drosophila Dystrophin, which acts with other components of the Dystrophin Associated Protein Complex to influence intercellular signalling in developing wing veins. Dev Biol 313: 519-532. CrossRef Medline

Crews ST (2010) Axon-glial interactions at the Drosophila CNS midline. Cell Adh Migr 4:67-71. CrossRef Medline

Crisp S, Evers JF, Fiala A, Bate M (2008) The development of motor coordination in Drosophila embryos. Development 135:3707-3717. CrossRef Medline

Dascenco D, Erfurth ML, Izadifar A, Song M, Sachse S, Bortnick R, Urwyler O, Petrovic M, Ayaz D, He H, Kise Y, Thomas F, Kidd T, Schmucker D (2015) Slit and receptor tyrosine phosphatase 69D confer spatial specificity to axon branching via Dscam1. Cell 162:1140-1154. CrossRef Medline

Dickson BJ, Zou Y (2010) Navigating intermediate targets: the nervous system midline. Cold Spring Harb Perspect Biol 2:a002055. CrossRef Medline

Di Costanzo S, Balasubramanian A, Pond HL, Rozkalne A, Pantaleoni C, Saredi S, Gupta VA, Sunu CM, Yu TW, Kang PB, Salih MA, Mora M, Gussoni E, Walsh CA, Manzini MC (2014) POMK mutations disrupt muscle development leading to a spectrum of neuromuscular presentations. Hum Mol Genet 23:5781-5792. CrossRef Medline

Dwyer CA, Katoh T, Tiemeyer M, Matthews RT (2015) Neurons and glia modify receptor protein-tyrosine phosphatase zeta (RPTPzeta)/phosphacan with cell-specific O-mannosyl glycans in the developing brain. J Biol Chem 290:10256-10273. CrossRef Medline

Fortier TM, Vasa PP, Woodard CT (2003) Orphan nuclear receptor betaFTZ-F1 is required for muscle-driven morphogenetic events at the prepupal-pupal transition in Drosophila melanogaster. Dev Biol 257: 153-165. CrossRef Medline

Freeze HH, Eklund EA, Ng BG, Patterson MC (2015) Neurological aspects of human glycosylation disorders. Annu Rev Neurosci 38:105-125. CrossRef Medline

Géminard C, González-Morales N, Coutelis JB, Noselli S (2014) The myosin ID pathway and left-right asymmetry in Drosophila. Genesis 52:471480. CrossRef Medline

Godfrey C, Foley AR, Clement E, Muntoni F (2011) Dystroglycanopathies: coming into focus. Curr Opin Genet Dev 21:278-285. CrossRef Medline

Gorczyca DA, Younger S, Meltzer S, Kim SE, Cheng L, Song W, Lee HY, Jan LY, Jan YN (2014) Identification of Ppk26, a DEG/ENaC channel functioning with Ppk1 in a mutually dependent manner to guide locomotion behavior in Drosophila. Cell Rep 9:1446-1458. CrossRef Medline

Grueber WB, Ye B, Yang CH, Younger S, Borden K, Jan LY, Jan YN (2007) Projections of Drosophila multidendritic neurons in the central nervous system: links with peripheral dendrite morphology. Development 134: 55-64. CrossRef Medline

Haines N, SeabrookeS, Stewart BA (2007) Dystroglycan and protein O-mannosyltransferases 1 and 2 are required to maintain integrity of Drosophila larval muscles. Mol Biol Cell 18:4721-4730. CrossRef Medline

Han C, Wang D, Soba P, Zhu S, Lin X, Jan LY, Jan YN (2012) Integrins regulate repulsion-mediated dendritic patterning of drosophila sensory neurons by restricting dendrites in a 2D space. Neuron 73:64-78. CrossRef Medline

Heckscher ES, Zarin AA, Faumont S, Clark MQ, Manning L, Fushiki A, Schneider-Mizell CM, Fetter RD, Truman JW, Zwart MF, Landgraf M, Cardona A, Lockery SR, Doe CQ (2015) Even-skipped(+) interneurons are core components of a sensorimotor circuit that maintains left-right symmetric muscle contraction amplitude. Neuron 88:314-329. CrossRef Medline

Hu H, Li J, Gagen CS, Gray NW, Zhang Z, Qi Y, Zhang P (2011) Conditional knockout of protein O-mannosyltransferase 2 reveals tissue-specific roles of O-mannosyl glycosylation in brain development. J Comp Neurol 519: 1320-1337. CrossRef Medline

Hughes CL, Thomas JB (2007) A sensory feedback circuit coordinates muscle activity in Drosophila. Mol Cell Neurosci 35:383-396. CrossRef Medline

Ichimiya T, Manya H, Ohmae Y, Yoshida H, Takahashi K, Ueda R, Endo T, Nishihara S (2004) The twisted-abdomen phenotype of drosophila POMT1 and POMT2 mutants coincides with their heterophilic protein O-mannosyltransferase activity. J Biol Chem 279:42638-42647. CrossRef Medline

Islam R, Nakamura M, Scott H, Repnikova E, Carnahan M, Pandey D, Caster C, Khan S, Zimmermann T, Zoran MJ, Panin VM (2013) The role of Drosophila cytidine monophosphate-sialic acid synthetase in the nervous system. J Neurosci 33:12306-12315. CrossRef Medline

Jacobs JR (2000) The midline glia of Drosophila: a molecular genetic model for the developmental functions of glia. Prog Neurobiol 62:475-508. CrossRef Medline

Kolodkin AL, Tessier-Lavigne M (2011) Mechanisms and molecules of neuronal wiring: a primer. Cold Spring Harb Perspect Biol 3:a001727. CrossRef Medline

Landgraf M, Sánchez-Soriano N, Technau GM, Urban J, Prokop A (2003) Charting the Drosophila neuropile: a strategy for the standardised characterisation of genetically amenable neurites. Dev Biol 260:207-225. CrossRef Medline

Leyten QH, Gabreels FJM, Renier WO, terLaak HJ (1996) Congenital muscular dystrophy: a review of the literature. Clin Neurol Neurosurg 98:267280. CrossRef Medline

Li J, Yu M, Feng G, Hu H, Li X (2011) Breaches of the pial basement membrane are associated with defective dentate gyrus development in mouse models of congenital muscular dystrophies. Neurosci Lett 505:19-24. CrossRef Medline

Lyalin D, Koles K, Roosendaal SD, Repnikova E, Van Wechel L, Panin VM (2006) The twisted gene encodes Drosophila protein O-mannosyltransferase 2 and genetically interacts with the rotated abdomen gene encoding Drosophila protein O-mannosyltransferase 1. Genetics 172:343-353. CrossRef Medline

Martin PT (2007) Congenital muscular dystrophies involving the O-mannose pathway. Curr Mol Med 7:417-425. CrossRef Medline

Martín-Blanco E, García-Bellido A (1996) Mutations in the rotated abdomen locus affect muscle development and reveal an intrinsic asymmetry in Drosophila. Proc Natl Acad Sci U S A 93:6048-6052. CrossRef Medline

Mercuri E, Muntoni F (2013) Muscular dystrophies. Lancet 381:845-860. CrossRef Medline

Moremen KW, Tiemeyer M, Nairn AV (2012) Vertebrate protein glycosylation: diversity, synthesis and function. Nat Rev Mol Cell Biol 13:448 462. CrossRef Medline

Morikawa RK, Kanamori T, Yasunaga K, Emoto K (2011) Different levels of the Tripartite motif protein, Anomalies in sensory axon patterning (Asap), regulate distinct axonal projections of Drosophila sensory neurons. Proc Natl Acad Sci U S A 108:19389-19394. CrossRef Medline

Nakamura N, Stalnaker SH, Lyalin D, Lavrova O, Wells L, Panin VM (2010a) Drosophila dystroglycan is a target of O-mannosyltransferase activity of two protein O-mannosyltransferases, rotated abdomen and twisted. Glycobiology 20:381-394. CrossRef Medline

Nakamura N, Lyalin D, Panin VM, Panin V (2010b) Pathogenic mechanisms associated with abnormal protein O-mannosylation: cell culture and Drosophila models. Semin Cell Dev Biol 21:622-630. CrossRef Medline

Neuhaus-Follini A, Bashaw GJ (2015) Crossing the embryonic midline: molecular mechanisms regulating axon responsiveness at an intermediate target. Wiley Interdiscip Rev Dev Biol 4:377-389. CrossRef Medline

Nolo R, Abbott LA, Bellen HJ (2000) Senseless, a Zn finger transcription factor, is necessary and sufficient for sensory organ development in Drosophila. Cell 102:349-362. CrossRef Medline

Organisti C, Hein I, Grunwald Kadow IC, Suzuki T (2015) Flamingo, a seven-pass transmembrane cadherin, cooperates with Netrin/Frazzled in Drosophila midline guidance. Genes Cells 20:50-67. CrossRef Medline

Panin VM, Wells L (2014) Protein O-mannosylation in metazoan organisms. Curr Protoc Protein Sci 75:Unit 12.12. CrossRef Medline 
Pereanu W, Spindler S, Im E, Buu N, Hartenstein V (2007) The emergence of patterned movement during late embryogenesis of Drosophila. Dev Neurobiol 67:1669-1685. CrossRef Medline

Ranganayakulu G, Schulz RA, Olson EN (1996) Wingless signaling induces nautilus expression in the ventral mesoderm of the Drosophila embryo. Dev Biol 176:143-148. CrossRef Medline

Robertson CW (1936) The metamorphosis of Drosophila melanogaster, including an accurately timed account for the principle morphological changes. J Morphol 59:351-399. CrossRef

Rothwell WF, Sullivan W (2000) Fluorescent analysis of Drosophila embryos. In: Drosophila protocols (Sullivan W, Ashburner M, Hawley RS, eds), pp. 141-158. Cold Spring Harbor, NY: Cold Spring Harbor Laboratory.

Schuster CM, Davis GW, Fetter RD, Goodman CS (1996) Genetic dissection of structural and functional components of synaptic plasticity. II. Fasciclin II controls presynaptic structural plasticity. Neuron 17:655667. CrossRef Medline

Shcherbata HR, Yatsenko AS, Patterson L, Sood VD, Nudel U, Yaffe D, Baker D, Ruohola-Baker H (2007) Dissecting muscle and neuronal disorders in a Drosophila model of muscular dystrophy. EMBO J 26:481-493. CrossRef Medline

Song W, Onishi M, Jan LY, Jan YN (2007) Peripheral multidendritic sensory neurons are necessary for rhythmic locomotion behavior in Drosophila larvae. Proc Natl Acad Sci U S A 104:5199-5204. CrossRef Medline

Suster ML, Bate M (2002) Embryonic assembly of a central pattern generator without sensory input. Nature 416:174-178. CrossRef Medline

Ueyama M, Akimoto Y, Ichimiya T, Ueda R, Kawakami H, Aigaki T, Nishihara S (2010) Increased apoptosis of myoblasts in Drosophila model for the Walker-Warburg syndrome. PLoS One 5:e11557. CrossRef Medline

Vester-Christensen MB, Halim A, Joshi HJ, Steentoft C, Bennett EP, Levery SB, Vakhrushev SY, Clausen H (2013) Mining the O-mannose glycoproteome reveals cadherins as major O-mannosylated glycoproteins. Proc Natl Acad Sci U S A 110:21018-21023. CrossRef Medline

Wairkar YP, Fradkin LG, Noordermeer JN, DiAntonio A (2008) Synaptic defects in a Drosophila model of congenital muscular dystrophy. J Neurosci 28:3781-3789. CrossRef Medline
Wells L (2013) The o-mannosylation pathway: glycosyltransferases and proteins implicated in congenital muscular dystrophy. J Biol Chem 288: 6930-6935. CrossRef Medline

Willer T, Prados B, Falcón-Pérez JM, Renner-Müller I, Przemeck GK, Lommel M, Coloma A, Valero MC, de Angelis MH, Tanner W, Wolf E, Strahl S, Cruces J (2004) Targeted disruption of the Walker-Warburg syndrome gene Pomt1 in mouse results in embryonic lethality. Proc Natl Acad Sci U S A 101:14126-14131. CrossRef Medline

Wong AM, Wang JW, Axel R (2002) Spatial representation of the glomerular map in the Drosophila protocerebrum. Cell 109:229-241. CrossRef Medline

Wu Z, Sweeney LB, Ayoob JC, Chak K, Andreone BJ, Ohyama T, Kerr R, Luo L, Zlatic M, Kolodkin AL (2011) A combinatorial semaphorin code instructs the initial steps of sensory circuit assembly in the Drosophila CNS. Neuron 70:281-298. CrossRef Medline

Yang L, Li R, Kaneko T, Takle K, Morikawa RK, Essex L, Wang X, Zhou J, Emoto K, Xiang Y, Ye B (2014) Trim9 regulates activity-dependent finescale topography in Drosophila. Curr Biol 24:1024-1030. CrossRef Medline

Yoshida-Moriguchi T, Campbell KP (2015) Matriglycan: a novel polysaccharide that links dystroglycan to the basement membrane. Glycobiology 25:702-713. CrossRef Medline

Zaffran S, Astier M, Gratecos D, Sémériva M (1997) The held out wings (how) Drosophila gene encodes a putative RNA-binding protein involved in the control of muscular and cardiac activity. Development 124:20872098. Medline

Zhong L, Hwang RY, Tracey WD (2010) Pickpocket is a DEG/ENaC protein required for mechanical nociception in drosophila larvae. Curr Biol 20: 429-434. CrossRef Medline

Zlatic M, Landgraf M, Bate M (2003) Genetic specification of axonal arbors: atonal regulates robo3 to position terminal branches in the Drosophila nervous system. Neuron 37:41-51. CrossRef Medline

Zlatic M, Li F, Strigini M, Grueber W, Bate M (2009) Positional cues in the Drosophila nerve cord: semaphorins pattern the dorsoventral axis. PLoS Biol 7:e1000135. CrossRef Medline 